IMA Journal of Numerical Analysis (2012) 32, 48-74

doi:10.1093/imanum/drq032

Advance Access publication on June 2, 2011

\title{
Second-order elliptic PDEs with discontinuous boundary data
}

\author{
PAUl Houston* \\ School of Mathematical Sciences, University of Nottingham, University Park, \\ Nottingham NG7 2RD, UK \\ ${ }^{*}$ Corresponding author: paul.houston@ nottingham.ac.uk \\ AND \\ THOMAS P. WiHLER \\ Mathematisches Institut, Universität Bern, Sidlerstrasse 5, CH-3012 Bern, Switzerland \\ wihler@math.unibe.ch
}

[Received on 17 December 2009; revised on 7 July 2010]

\begin{abstract}
We consider the weak formulation of a linear elliptic model problem with discontinuous Dirichlet boundary conditions. Since such problems are typically not well defined in the standard $H^{1}-H^{1}$ setting we introduce a suitable saddle point formulation in terms of weighted Sobolev spaces. Furthermore, we discuss the numerical solution of such problems. Specifically, we employ an $h p$-discontinuous Galerkin method and derive (enhanced) $L^{2}$-norm upper and local lower a posteriori error bounds. Numerical experiments demonstrate the effectiveness of the proposed error indicator in both the $h$ - and the $h p$-version setting. Indeed, in the latter case, exponential convergence of the error is attained as the mesh is adaptively refined.
\end{abstract}

Keywords: second-order elliptic PDE; discontinuous Dirichlet boundary conditions; inf-sup condition; $h p$-discontinuous Galerkin FEM; $L^{2}$-norm a posteriori error analysis; exponential convergence.

\section{Introduction}

On a bounded polygonal domain $\Omega \subset \mathbb{R}^{2}$ with straight edges and connected boundary $\Gamma=\partial \Omega$, we consider the linear diffusion-reaction problem

$$
\begin{aligned}
-\Delta u+c u=f & \text { in } \Omega, \\
u=g & \text { on } \Gamma,
\end{aligned}
$$

where $c \in L^{\infty}(\Omega)$ is a non-negative function, $f \in L^{2}(\Omega)$ and $g \in L^{2}(\Gamma)$ is a possibly discontinuous function on $\Gamma$ whose precise regularity will be specified later. Throughout the paper we shall use the following notation. For a domain $D \subset \mathbb{R}^{n}(n=1$ or $n=2)$ we denote by $L^{2}(D)$ the space of all square-integrable functions on $D$, with norm $\|\cdot\|_{0, D}$. Furthermore, for an integer $k \in \mathbb{N}_{0}$, we let $H^{k}(D)$ be the usual Sobolev space of order $k$ on $D$, with norm $\|\cdot\|_{k, D}$ and seminorm $|\cdot|_{k, D}$. The space $\stackrel{\circ}{H}^{1}(\Omega)$ is defined as the subspace of $H^{1}(\Omega)$ consisting of functions with zero trace on $\Gamma$.

Several variational formulations for elliptic problems with discontinuous Dirichlet boundary conditions exist. We mention the very weak formulation which is to find a solution $u \in L^{2}(\Omega)$ such that

$$
-\int_{\Omega} u \Delta v \mathrm{~d} \boldsymbol{x}+\int_{\Omega} c u v \mathrm{~d} \boldsymbol{x}=\int_{\Omega} f v \mathrm{~d} \boldsymbol{x}-\int_{\Gamma} g \nabla v \cdot \mathbf{n} \mathrm{d} s
$$

(c) The author 2011. Published by Oxford University Press on behalf of the Institute of Mathematics and its Applications. All rights reserved. 
for any $v \in H^{2}(\Omega) \cap \stackrel{\circ}{H}^{1}(\Omega)$, where $\mathbf{n}$ denotes the unit outward normal vector to the boundary $\Gamma$. It is based on twofold integration by parts of (1.1) and incorporates the Dirichlet boundary data in a natural way. On the other hand, however, the numerical solution by means of a conforming finite element discretization would require continuously differentiable test functions. In order to avoid this problem the following saddle point formulation can be used (see Nečas, 1962): provided that $g \in H^{1 / 2-\varepsilon}(\Gamma)$, for some $\varepsilon \in[0,1 / 2)$, find $u \in H^{1-\varepsilon}(\Omega)$ with $\left.u\right|_{\Gamma}=g$ such that

$$
\int_{\Omega} \nabla u \cdot \nabla v \mathrm{~d} \boldsymbol{x}+\int_{\Omega} c u v \mathrm{~d} \boldsymbol{x}=\int_{\Omega} f v \mathrm{~d} \boldsymbol{x}
$$

for all $v \in H^{1+\varepsilon}(\Omega) \cap \stackrel{\circ}{H}^{1}(\Omega)$. We note that the bilinear form on the left-hand side is formally symmetric and corresponds to the standard form for the Poisson equation. For results dealing with related finite element approximations we refer to Babuška (1971).

In the present paper a new variational formulation for (1.1)-(1.2) is presented and analysed. Here the emphasis shall be on Dirichlet boundary conditions that may exhibit (isolated) discontinuities and are essentially continuous otherwise. The formulation in this article is closely related to the saddle point formulation (1.3), however, it features Sobolev spaces that describe the local singularities in the analytical solution resulting from the discontinuities in the boundary data in a more specific way. More precisely, weighted Sobolev spaces that have been used in the context of regularity statements for second-order elliptic boundary value problems, see, e.g., Babuška \& Guo $(1988,1989)$ and Guo \& Schwab (2006), will be used. The idea of applying weights for problems with discontinuous boundary data has been employed previously in Bernardi \& Karageorghis (1999). For the formulation in the current paper we will establish well-posedness of the weak formulation in terms of an appropriate inf-sup condition.

In order to discretize the underlying partial differential equation (PDE) problem we employ a framework that allows possible singularities in the solution to be resolved efficiently (see, e.g., Babuška et al., 1979; Guo \& Babuška, 1986a,b; Schwab, 1998; Nicaise, 2000 for results on the approximation of singularities in weighted Sobolev spaces). Specifically, in this paper, we shall exploit the $h p$-version of the symmetric interior penalty discontinuous Galerkin (DG) finite element method, cf. Arnold et al. (2001), and the references cited therein. DG methods are ideally suited for realizing $h p$-adaptivity for secondorder boundary value problems, an advantage that has been noted early on in the recent development of these methods; see, for example, Baumann \& Oden (1999), Rivière et al. (1999), Cockburn et al. (2000), Perugia \& Schötzau (2002), Wihler et al. (2003), Houston et al. (2002, 2007, 2008), Stamm \& Wihler (2010) and the references therein. Indeed, working with discontinuous finite element spaces easily facilitates the use of variable polynomial degrees and local mesh refinement techniques on possibly irregularly refined meshes - the two key ingredients for $h p$-adaptive algorithms. A further advantage of interior penalty DG formulations is that they incorporate Dirichlet boundary conditions in a natural way irrespective of their smoothness (in fact, $L^{1}$-regularity is sufficient for well-posedness). With this in mind, we shall derive computable upper and local lower a posteriori bounds for the error measured in terms of an enhanced $L^{2}$-norm on $\Omega$. On the basis of the resulting computable error indicators, adaptive $h$ - and $h p$-mesh adaptation strategies will be investigated for a model second-order elliptic PDE with discontinuous boundary conditions. In particular, we shall show numerically that exploiting $h p$-refinement leads to exponential convergence of the (enhanced) $L^{2}$-norm of the error as the finite element space is enriched.

The article is organized as follows. In Section 2 the new variational formulation of (1.1)-(1.2) will be presented. In addition, its well-posedness will be proved. Then, in Section 3, we will briefly review $h p$-version DG discretizations for the Laplace operator and derive $L^{2}$-norm a posteriori error estimates. 
Additionally, the performance of the corresponding local error indicators is shown with a number of numerical experiments within an $h$ - and $h p$-version adaptive framework. Finally, a few concluding remarks are made in Section 4.

\section{Variational formulation}

\subsection{Weighted Sobolev spaces}

Let $\mathcal{A}=\left\{A_{i}\right\}_{i=1}^{M} \subset \Gamma, A_{i} \neq A_{j}$ for $i \neq j$, be a finite set of points on the boundary of the polygonal domain $\Omega$ which are numbered in a counter clockwise direction along $\Gamma$; the points in $\mathcal{A}$ will signify the locations of the discontinuities in the Dirichlet boundary condition $g$ in (1.2). Furthermore, we denote by $\Gamma_{i} \subset \Gamma, i=1,2, \ldots, M$, the (open) subset of $\Gamma$, which connects the two points $A_{i}$ and $A_{i+1}$; here we set $A_{M+1}=A_{1}$. Moreover, let $\omega_{i} \in(0,2 \pi]$ signify the interior angle of the polygon $\Omega$ at $A_{i}$. To each $A_{i} \in \mathcal{A}, i=1,2, \ldots, M$, we associate a weight $\alpha_{i} \in \mathbb{R}$. These numbers are stored in a weight vector

$$
\boldsymbol{\alpha}=\left(\alpha_{1}, \alpha_{2}, \ldots, \alpha_{M}\right) \in \mathbb{R}^{M} .
$$

Moreover, for any number $k \in \mathbb{R}$, we use the notation $k \boldsymbol{\alpha}=\left(k \alpha_{1}, k \alpha_{2}, \ldots, k \alpha_{M}\right)$ and $\boldsymbol{\alpha}+k=$ $\left(\alpha_{1}+k, \alpha_{2}+k, \ldots, \alpha_{M}+k\right)$. Furthermore, for a fixed number

$$
\eta>0
$$

we introduce the following weight function on $\Omega$ :

$$
\Phi_{\boldsymbol{\alpha}}(\boldsymbol{x})=\prod_{i=1}^{M} r_{i}(\boldsymbol{x})^{\alpha_{i}}, \quad r_{i}(\boldsymbol{x})=\min \left\{\eta^{-1}\left|\boldsymbol{x}-A_{i}\right|, 1\right\} .
$$

Here we assume that $\eta$ is small enough, so that the open sectors

$$
S_{i}=\left\{x \in \Omega:\left|x-A_{i}\right|<\eta\right\}, \quad i=1,2, \ldots, M,
$$

do not intersect, i.e., $\bar{S}_{i} \cap \bar{S}_{j}=\emptyset$ if $i \neq j$. There holds, for $x \in \Omega$, that

$$
r_{i}(\boldsymbol{x})= \begin{cases}\eta^{-1}\left|\boldsymbol{x}-A_{i}\right| & \text { if } \boldsymbol{x} \in S_{i}, \\ 1 & \text { if } \boldsymbol{x} \in \Omega \backslash S_{i},\end{cases}
$$

and $r_{i} \in C^{0}(\Omega), i=1,2, \ldots, M$. Furthermore, setting

$$
\mathscr{S}=\bigcup_{i=1}^{M} S_{i}, \quad \Omega_{0}=\Omega \backslash \overline{\mathscr{S}},
$$

we have

$$
\Phi_{\alpha}= \begin{cases}r_{i}^{\alpha_{i}} & \text { if } \boldsymbol{x} \in S_{i} \text { for some } i=1,2, \ldots M \\ 1 & \text { if } \boldsymbol{x} \in \Omega_{0}\end{cases}
$$


Note that $\Phi_{\boldsymbol{\alpha}}$ is continuous on $\Omega$. Furthermore, for $\boldsymbol{\alpha}_{1}, \boldsymbol{\alpha}_{2} \in \mathbb{R}^{M}$, we have

$$
\Phi_{\boldsymbol{\alpha}_{1}+\boldsymbol{\alpha}_{2}}=\Phi_{\boldsymbol{\alpha}_{1}} \Phi_{\boldsymbol{\alpha}_{2}}, \quad \Phi_{\alpha}^{-1}=\Phi_{-\alpha}
$$

Then, for any integers $m \geqslant l \geqslant 0$, we define the weighted Sobolev spaces $H_{\boldsymbol{\alpha}}^{m, l}(\Omega)$ as the completion of the space $C^{\infty}(\bar{\Omega})$ with respect to the weighted Sobolev norms

$$
\begin{aligned}
\|u\|_{H_{a}^{m, l}(\Omega)}^{2} & =\|u\|_{l-1, \Omega}^{2}+\sum_{k=l}^{m}|u|_{H_{\alpha}^{k, l}(\Omega)}^{2}, \quad l \geqslant 1, \\
\|u\|_{H_{\alpha}^{m, 0}(\Omega)}^{2} & =\sum_{k=0}^{m}|u|_{H_{a}^{k, 0}(\Omega)}^{2} .
\end{aligned}
$$

Here

$$
|u|_{H_{\boldsymbol{\alpha}}^{k, l}(\Omega)}^{2}=\sum_{|\lambda|=k}\left\|\Phi_{\boldsymbol{\alpha}+k-l}\left|\mathrm{D}^{\lambda} u\right|\right\|_{0, \Omega}^{2}
$$

is the $H_{\alpha}^{k, l}$-seminorm in $\Omega$, where

$$
\mathrm{D}^{\lambda} u=\frac{\partial^{|\lambda|} u}{\partial x_{1}^{\lambda_{1}} \partial x_{2}^{\lambda_{2}}},
$$

with $\lambda=\left(\lambda_{1}, \lambda_{2}\right) \in \mathbb{N}_{0}^{2}$ and $|\lambda|=\lambda_{1}+\lambda_{2}$.

In addition, for $m \geqslant l \geqslant 1$, let us define the space $H_{\boldsymbol{\alpha}}^{m-\frac{1}{2}, l-\frac{1}{2}}(\Gamma)$ as the trace space of $H_{\boldsymbol{\alpha}}^{m, l}(\Omega)$, equipped with the norm

$$
\|u\|_{H_{\alpha}^{m-\frac{1}{2}, l-\frac{1}{2}}(\Gamma)}=\inf _{\substack{\left.v \in H_{\alpha}^{m, l}(\Omega) \\ v\right|_{\partial \Omega}=u}}\|v\|_{H_{a}^{m, l}(\Omega)} .
$$

Moreover, we denote by $\stackrel{\circ}{\alpha}_{\alpha}^{m, l}(\Omega)$ the subspace of $H_{\alpha}^{m, l}(\Omega)$ consisting of functions with zero trace on $\Gamma$.

\subsection{Inequalities in $H_{\alpha}^{1,1}(\Omega)$}

In order to describe the well-posedness of (1.1)-(1.2) the weighted Sobolev space $H_{\alpha}^{1,1}(\Omega)$ will play an important role. In the sequel we shall collect a few inequalities that will be used for the analysis in this paper.

Lemma 2.1 Let $I=(a, b) \subset \mathbb{R}, a<b$, be an open interval. Then there holds the Poincaré-Friedrichs inequality

$$
\int_{a}^{b} \phi(x)^{2} \mathrm{~d} x \leqslant \frac{(b-a)^{2}}{\pi^{2}} \int_{a}^{b}\left(\phi^{\prime}(x)\right)^{2} \mathrm{~d} \boldsymbol{x}
$$

for all $\phi \in H^{1}(a, b)$ with $\phi(a)=\phi(b)=0$.

Proof. The bound follows from Hardy et al. (1952, Theorem 257) and a scaling argument.

Applying the previous lemma we shall prove the following result. 
LEMMA 2.2 Consider a sector $S=\left\{(r, \theta): 0<r<R, \theta_{0}<\theta<\theta_{1}\right\} \subset \mathbb{R}^{2}$, where $(r, \theta)$ denote polar coordinates in $\mathbb{R}^{2}$, and $R>0,0 \leqslant \theta_{0}<\theta_{1} \leqslant 2 \pi$ are constants. Furthermore, let $u \in L^{2}(S)$ with $\left\|r^{\alpha} \nabla u\right\|_{0, S}<\infty$ for some $\alpha \in[0,1)$, and $\left.u\right|_{\partial S_{<}}=0$, where $\partial S_{<}=\left\{(r, \theta): 0<r<R, \theta \in\left\{\theta_{0}, \theta_{1}\right\}\right\}$. Then there holds

$$
\int_{S} r^{2 \alpha-2} u(\boldsymbol{x})^{2} \mathrm{~d} \boldsymbol{x} \leqslant \frac{\left(\theta_{1}-\theta_{0}\right)^{2}}{\pi^{2}} \int_{S} r^{2 \alpha}|\nabla u|^{2} \mathrm{~d} \boldsymbol{x} .
$$

Proof. Using integration in polar coordinates we get

$$
\int_{S} r^{2 \alpha-2} u(\boldsymbol{x})^{2} \mathrm{~d} \boldsymbol{x}=\int_{0}^{R} r^{2 \alpha-1} \int_{\theta_{0}}^{\theta_{1}} u^{2} \mathrm{~d} \theta \mathrm{d} r .
$$

Then since for any $r \in(0, R)$ there holds $u\left(r, \theta_{0}\right)=u\left(r, \theta_{1}\right)=0$ we can apply Lemma 2.1. This implies

$$
\int_{\theta_{0}}^{\theta_{1}} u^{2} \mathrm{~d} \theta \leqslant \frac{\left(\theta_{1}-\theta_{0}\right)^{2}}{\pi^{2}} \int_{\theta_{0}}^{\theta_{1}}\left|\partial_{\theta} u\right|^{2} \mathrm{~d} \theta, \quad 0<r<R .
$$

Furthermore, noting that $\left|\partial_{\theta} u\right| \leqslant r\left|\nabla_{x} u\right|$, we obtain

$$
\int_{\theta_{0}}^{\theta_{1}} u^{2} \mathrm{~d} \theta \leqslant \frac{\left(\theta_{1}-\theta_{0}\right)^{2}}{\pi^{2}} r^{2} \int_{\theta_{0}}^{\theta_{1}}\left|\nabla_{x} u\right|^{2} \mathrm{~d} \theta, \quad 0<r<R .
$$

Inserting this estimate into (2.5) leads to

$$
\int_{S} r^{2 \alpha-2} u(x)^{2} \mathrm{~d} \boldsymbol{x} \leqslant \frac{\left(\theta_{1}-\theta_{0}\right)^{2}}{\pi^{2}} \int_{0}^{R} r^{2 \alpha+1} \int_{\theta_{0}}^{\theta_{1}}\left|\nabla_{\boldsymbol{x}} u\right|^{2} \mathrm{~d} \theta \mathrm{d} r .
$$

Changing back to Cartesian coordinates $\boldsymbol{x}$ completes the proof.

LEMMA 2.3 Given a weight vector $\boldsymbol{\alpha} \in[0,1)^{M}$. Then there holds

$$
\left\|\Phi_{-\alpha} u\right\|_{0, \Omega} \leqslant C\|u\|_{1, \Omega}
$$

for any $u \in H^{1}(\Omega)$, where the constant $C>0$ only depends on $\boldsymbol{\alpha}$ and $\Omega$.

Proof. Let $S_{i}, i=1,2, \ldots, M$, be the (sufficiently small) sectors from (2.3). Then we recall the property (2.4) to write

$$
\left\|\Phi_{-\alpha} u\right\|_{0, \Omega}^{2}=\|u\|_{0, \Omega_{0}}^{2}+\left\|\Phi_{-\alpha} u\right\|_{0, \mathscr{S}}^{2}=\|u\|_{0, \Omega_{0}}^{2}+\sum_{i=1}^{M}\left\|r_{i}^{-\alpha_{i}} u\right\|_{0, S_{i}}^{2}
$$

If, for some $1 \leqslant i \leqslant M$, we have that $\alpha_{i}>0$, then

$$
\left\|r_{i}^{-\alpha_{i}} u\right\|_{0, S_{i}}^{2} \leqslant C\left(\|u\|_{0, S_{i}}^{2}+\left\|r_{i}^{1-\alpha_{i}} \nabla u\right\|_{0, S_{i}}^{2}\right) \leqslant C\|u\|_{1, S_{i}}^{2}
$$

this follows from expressing the norms in terms of polar coordinates and from applying Hardy et al. (1952, Theorem 330). Inserting this into (2.6) gives the desired inequality. 
Lemma 2.4 Consider a function $u \in \stackrel{\circ}{H}_{\alpha}^{1,1}(\Omega)$, where $\alpha_{i} \in[0,1), i=1,2, \ldots, M$. Then there holds

$$
\left\|\left|\nabla\left(\Phi_{\boldsymbol{\alpha}}\right)\right| u\right\|_{0, \Omega} \leqslant \frac{1}{\pi} \max _{1 \leqslant i \leqslant M} \alpha_{i} \omega_{i}|u|_{H_{\alpha}^{1,1}(\Omega)} .
$$

Proof. Let $S_{i}, i=1,2, \ldots, M$, be the (sufficiently small) sectors from (2.3). Then, due to (2.4), we have

$$
\left|\nabla\left(\Phi_{\boldsymbol{\alpha}}\right)\right|= \begin{cases}\left|\nabla\left(r_{i}^{\alpha_{i}}\right)\right|=\alpha_{i} \eta^{-1} r_{i}^{\alpha_{i}-1} & \text { if } \boldsymbol{x} \in S_{i} \text { for some } i=1,2, \ldots, M, \\ 0 & \text { if } \boldsymbol{x} \in \Omega_{0} .\end{cases}
$$

Hence,

$$
\int_{\Omega}\left|\nabla\left(\Phi_{\boldsymbol{\alpha}}\right)\right|^{2} u^{2} \mathrm{~d} \boldsymbol{x}=\eta^{-2} \sum_{i=1}^{M} \alpha_{i}^{2} \int_{S_{i}} r_{i}^{2 \alpha_{i}-2} u^{2} \mathrm{~d} \boldsymbol{x} .
$$

Then, applying Lemma 2.2, we have

$$
\int_{S_{i}} r_{i}^{2 \alpha_{i}-2} u^{2} \mathrm{~d} \boldsymbol{x} \leqslant \eta^{2} \frac{\omega_{i}^{2}}{\pi^{2}} \int_{S_{i}} r_{i}^{2 \alpha_{i}}|\nabla u|^{2} \mathrm{~d} \boldsymbol{x}
$$

Thus,

$$
\int_{\Omega}\left|\nabla\left(\Phi_{\boldsymbol{\alpha}}\right)\right|^{2} u^{2} \mathrm{~d} \boldsymbol{x} \leqslant \sum_{i=1}^{M} \frac{\alpha_{i}^{2} \omega_{i}^{2}}{\pi^{2}} \int_{S_{i}} r_{i}^{2 \alpha_{i}}|\nabla u|^{2} \mathrm{~d} \boldsymbol{x} \leqslant \frac{\max _{1 \leqslant i \leqslant M}\left(\alpha_{i}^{2} \omega_{i}^{2}\right)}{\pi^{2}} \int_{\Omega} \Phi_{\alpha}^{2}|\nabla u|^{2} \mathrm{~d} \boldsymbol{x}
$$

as required.

Furthermore, there holds the following Poincaré-Friedrichs inequality.

Lemma 2.5 Consider a weight vector $\boldsymbol{\alpha} \in[0,1)^{M}$ and $\gamma \subseteq \Gamma$ with $\int_{\gamma} \mathrm{d} s>0$. Then there exists a constant $C>0$ depending only on $\gamma, \Omega$ and $\boldsymbol{\alpha}$ such that

$$
\|u\|_{0, \Omega} \leqslant C|u|_{H_{\alpha}^{1,1}(\Omega)}
$$

for all functions $u \in H_{\alpha}^{1,1}(\Omega)$ with $\left.u\right|_{\gamma}=0$ (in the trace sense). In particular, we have that $|\cdot|_{H_{\alpha}^{1,1}(\Omega)}$ is a norm on $\stackrel{\circ}{H}_{\alpha}^{1,1}(\Omega)$.

Proof. We first note that the embedding $W^{1,1}(\Omega) \hookrightarrow L^{2}(\Omega)$ is continuous for Lipschitz polygons in $\mathbb{R}^{2}$ (cf., e.g., Adams \& Fournier, 2003, Theorem 4.12). Hence, there exists a constant $C>0$ depending on $\Omega$ such that

$$
\|u\|_{0, \Omega} \leqslant C\|u\|_{W^{1,1}(\Omega)} .
$$

Moreover, applying the Poincaré-Friedrichs inequality in $W^{1,1}(\Omega)$, it follows that

$$
\|u\|_{0, \Omega} \leqslant C\|u\|_{W^{1,1}(\Omega)} \leqslant C^{\prime}\|\nabla u\|_{L^{1}(\Omega)}
$$

for a constant $C^{\prime}>0$ depending on $\gamma$ and $\Omega$. Therefore, using Hölder's inequality, we obtain

$$
\|u\|_{0, \Omega} \leqslant C^{\prime} \int_{\Omega}|\nabla u| \mathrm{d} \mathbf{x} \leqslant C^{\prime}\left(\int_{\Omega} \Phi_{\alpha}^{-2} \mathrm{~d} \boldsymbol{x}\right)^{\frac{1}{2}}\left(\int_{\Omega} \Phi_{\alpha}^{2}|\nabla u|^{2} \mathrm{~d} \boldsymbol{x}\right)^{\frac{1}{2}} .
$$


Then employing (2.4) yields

$$
\int_{\Omega} \Phi_{\alpha}^{-2} \mathrm{~d} \boldsymbol{x}=\sum_{i=1}^{M} \int_{S_{i}} r_{i}^{-2 \alpha_{i}} \mathrm{~d} \boldsymbol{x}+\int_{\Omega_{0}} 1 \mathrm{~d} \boldsymbol{x}
$$

and using integration in polar coordinates it follows that the above integrals are all bounded for $\alpha_{i}<1$, $i=1,2, \ldots, M$. This completes the proof.

To close this section we shall prove the following Green type formulae.

LEMMA 2.6 Let $\boldsymbol{\alpha} \in[0,1)^{M}$ be a weight vector and consider two functions $u \in H_{\alpha}^{1,1}(\Omega)$ and $\phi \in$ $H^{2}(\Omega)$. In addition, suppose that the trace of $\left.u\right|_{\Gamma} \in L^{2}(\Gamma)$. Then

$$
\int_{\Omega} \Delta \phi u \mathrm{~d} \boldsymbol{x}=\int_{\Gamma}(\nabla \phi \cdot \mathrm{n}) u \mathrm{~d} s-\int_{\Omega} \nabla \phi \cdot \nabla u \mathrm{~d} \boldsymbol{x}
$$

holds true, where $\mathbf{n}$ denotes the outward unit vector to $\Gamma$.

Proof. Due to the density of $C^{\infty}(\bar{\Omega})$ in $H_{\alpha}^{1,1}(\Omega)$ we can choose a sequence $\left\{u_{n}\right\}_{n \geqslant 0} \subset C^{\infty}(\bar{\Omega})$ such that $\lim _{n \rightarrow \infty}\left\|u-u_{n}\right\|_{H_{a}^{1,1}(\Omega)}=0$. Then, using Green's formula for smooth functions, we have

$$
\int_{\Omega} \Delta \phi u_{n} \mathrm{~d} \boldsymbol{x}=\int_{\Gamma}(\nabla \phi \cdot \boldsymbol{n}) u_{n} \mathrm{~d} s-\int_{\Omega} \nabla \phi \cdot \nabla u_{n} \mathrm{~d} \boldsymbol{x}
$$

for any function $\phi \in C^{\infty}(\bar{\Omega})$. Furthermore, there holds

$$
\left|\int_{\Omega} \Delta \phi\left(u_{n}-u\right) \mathrm{d} \boldsymbol{x}\right| \leqslant\|\phi\|_{2, \Omega}\left\|u-u_{n}\right\|_{0, \Omega} \stackrel{n \rightarrow \infty}{\longrightarrow} 0
$$

and, using Lemma 2.3,

$$
\begin{aligned}
\left|\int_{\Omega} \nabla \phi \cdot \nabla\left(u_{n}-u\right) \mathrm{d} \boldsymbol{x}\right| & \leqslant\left\|\Phi_{-\alpha} \nabla \phi\right\|_{0, \Omega}\left\|\Phi_{\boldsymbol{\alpha}} \nabla\left(u-u_{n}\right)\right\|_{0, \Omega} \\
& \leqslant C\|\phi\|_{2, \Omega}\left\|u-u_{n}\right\|_{H_{\boldsymbol{\alpha}}^{1,1}(\Omega)} \stackrel{n \rightarrow \infty}{\longrightarrow} 0 .
\end{aligned}
$$

Furthermore, applying the trace theorem in $W^{1,1}(\Omega)$ yields

$$
\begin{aligned}
\left|\int_{\Gamma}(\nabla \phi \cdot \boldsymbol{n})\left(u_{n}-u\right) \mathrm{d} s\right| & \leqslant \sup _{\bar{\Omega}}|\nabla \phi|\left\|u-u_{n}\right\|_{L^{1}(\Gamma)} \\
& \leqslant C \sup _{\bar{\Omega}}|\nabla \phi|\left(\left\|u-u_{n}\right\|_{L^{1}(\Omega)}+\left\|\nabla\left(u-u_{n}\right)\right\|_{L^{1}(\Omega)}\right) \\
& \leqslant C \sup _{\bar{\Omega}}|\nabla \phi|\left(\left\|u-u_{n}\right\|_{0, \Omega}+\left\|\Phi_{-\alpha}\right\|_{0, \Omega}\left\|\Phi_{\alpha} \nabla\left(u-u_{n}\right)\right\|_{0, \Omega}\right) \\
& \leqslant C \sup _{\bar{\Omega}}|\nabla \phi|\left\|u-u_{n}\right\|_{H_{a}^{1,1}(\Omega)} \stackrel{n \rightarrow \infty}{\longrightarrow} 0 .
\end{aligned}
$$

This implies the identity (2.9) for $u \in H_{\alpha}^{1,1}(\Omega)$ and $\phi \in C^{\infty}(\bar{\Omega})$. 
For $\phi \in H^{2}(\Omega)$ the density of $C^{\infty}(\bar{\Omega})$ in $H^{2}(\Omega)$ guarantees the existence of a sequence $\left\{\phi_{n}\right\}_{n \geqslant 0} \subset$ $C^{\infty}(\bar{\Omega})$ with $\lim _{n \rightarrow \infty}\left\|\phi_{n}-\phi\right\|_{2, \Omega}=0$. Then

$$
\int_{\Omega} \Delta \phi_{n} u \mathrm{~d} \boldsymbol{x}=\int_{\Gamma}\left(\nabla \phi_{n} \cdot \boldsymbol{n}\right) u \mathrm{~d} s-\int_{\Omega} \nabla \phi_{n} \cdot \nabla u \mathrm{~d} \boldsymbol{x}
$$

for all $u \in H_{\boldsymbol{\alpha}}^{1,1}(\Omega)$. Similarly, as before, we have

$$
\left|\int_{\Omega} \Delta\left(\phi_{n}-\phi\right) u \mathrm{~d} \boldsymbol{x}\right| \leqslant\left\|\phi_{n}-\phi\right\|_{2, \Omega}\|u\|_{0, \Omega} \stackrel{n \rightarrow \infty}{\longrightarrow} 0,
$$

and, with Lemma 2.3,

$$
\begin{aligned}
\left|\int_{\Omega} \nabla\left(\phi_{n}-\phi\right) \cdot \nabla u \mathrm{~d} \boldsymbol{x}\right| & \leqslant\left\|\Phi_{-\boldsymbol{\alpha}} \nabla\left(\phi_{n}-\phi\right)\right\|_{0, \Omega}\left\|\Phi_{\boldsymbol{\alpha}} \nabla u\right\|_{0, \Omega} \\
& \leqslant\left\|\phi_{n}-\phi\right\|_{2, \Omega}\|u\|_{H_{\boldsymbol{\alpha}}^{1,1}(\Omega)} \stackrel{n \rightarrow \infty}{\longrightarrow} 0 .
\end{aligned}
$$

Moreover, using the trace theorem again, we obtain

$$
\begin{aligned}
\left|\int_{\Gamma}\left(\nabla\left(\phi_{n}-\phi\right) \cdot \boldsymbol{n}\right) u \mathrm{~d} s\right| & \leqslant\left\|\nabla\left(\phi_{n}-\phi\right)\right\|_{L^{2}(\Gamma)}\|u\|_{L^{2}(\Gamma)} \\
& \leqslant C\left\|\phi_{n}-\phi\right\|_{2, \Omega}\|u\|_{L^{2}(\Gamma)} \stackrel{n \rightarrow \infty}{\longrightarrow} 0 .
\end{aligned}
$$

This completes the proof.

Lemma 2.7 Let $\boldsymbol{\alpha} \in[0,1)^{M}$, and $\Omega_{0} \subseteq \Omega$ a connected subset with Lipschitz boundary. Furthermore, consider $u \in H_{\alpha}^{1,1}\left(\Omega_{0}\right)$ with $\Delta u \in L^{2}\left(\Omega_{0}\right)$, and $v \in W^{1, \infty}\left(\Omega_{0}\right)$ with $\left.v\right|_{\Gamma}=0$. Then there holds

$$
\int_{\Omega_{0}} v \Delta u \mathrm{~d} \boldsymbol{x}+\int_{\Omega_{0}} \nabla u \cdot \nabla v \mathrm{~d} \boldsymbol{x}=0
$$

Here the space $H_{\boldsymbol{\alpha}}^{1,1}\left(\Omega_{0}\right)$ is defined as the restriction of $H_{\boldsymbol{\alpha}}^{1,1}(\Omega)$ to $\Omega_{0}$.

Proof. This follows again by density and from the fact that $W^{1, \infty}\left(\Omega_{0}\right) \hookrightarrow H_{-\alpha}^{1,1}\left(\Omega_{0}\right)$ continuously for $\boldsymbol{\alpha} \in[0,1)^{M} ;$ in particular, all integrals are well defined.

\subsection{Weak formulation}

The aim of this section is to introduce a weak formulation for the boundary value problem (1.1)-(1.2) and to discuss its well-posedness.

Let $g \in H_{\boldsymbol{\alpha}}^{1 / 2,1 / 2}(\Gamma)$ in (1.2), where $\boldsymbol{\alpha}$ is the weight vector from (2.1) with $\alpha_{i} \in[0,1), i=$ $1,2, \ldots, M$. Then we call $u \in H_{\alpha}^{1,1}(\Omega)$ with $\left.u\right|_{\Gamma}=g$ a weak solution of (1.1)-(1.2) if

$$
\int_{\Omega} \nabla u \cdot \nabla v \mathrm{~d} \boldsymbol{x}+\int_{\Omega} c u v \mathrm{~d} \boldsymbol{x}=\int_{\Omega} f v \mathrm{~d} \boldsymbol{x} \quad \forall v \in \stackrel{H}{-\alpha}_{-\alpha}^{1,1}(\Omega) .
$$

Writing the solution in the form $u=u_{0}+G$, where $u_{0} \in \stackrel{\circ}{H}_{\alpha}^{1,1}(\Omega)$ and $G \in H_{\alpha}^{1,1}(\Omega)$ is a lifting of the boundary data $g$, i.e., $\left.G\right|_{\Gamma}=g$, there holds

$$
\int_{\Omega} \nabla u_{0} \cdot \nabla v \mathrm{~d} \boldsymbol{x}+\int_{\Omega} c u_{0} v \mathrm{~d} \boldsymbol{x}=\int_{\Omega} f v \mathrm{~d} \boldsymbol{x}-\int_{\Omega} \nabla G \cdot \nabla v \mathrm{~d} \boldsymbol{x}-\int_{\Omega} c G v \mathrm{~d} \boldsymbol{x} \quad \forall v \in \stackrel{\circ}{H}_{-\boldsymbol{\alpha}}^{1,1}(\Omega) .
$$


We note that this is a saddle point formulation on $\stackrel{\circ}{H}_{\boldsymbol{\alpha}}^{1,1}(\Omega) \times \stackrel{\circ}{H}_{-\boldsymbol{\alpha}}^{1,1}(\Omega)$. Its well-posedness will be discussed in the following.

We first show that the bilinear form

$$
a(u, v)=\int_{\Omega} \nabla u \cdot \nabla v \mathrm{~d} \boldsymbol{x}+\int_{\Omega} c u v \mathrm{~d} \boldsymbol{x}
$$

and the linear functional

$$
\ell(v)=\int_{\Omega} f v \mathrm{~d} \boldsymbol{x}-\int_{\Omega} \nabla G \cdot \nabla v \mathrm{~d} \boldsymbol{x}-\int_{\Omega} c G v \mathrm{~d} \boldsymbol{x}=\int_{\Omega} f v \mathrm{~d} \boldsymbol{x}-a(G, v)
$$

are continuous. Here we suppose that the lifting $G$ is chosen such that

$$
\|G\|_{H_{\alpha}^{1,1}(\Omega)} \leqslant C\|g\|_{H_{\alpha}^{1 / 2,1 / 2}(\Gamma)}
$$

for some fixed constant $C>1$ independent of $g$.

Proposition 2.8 Let $\boldsymbol{\alpha} \in[0,1)^{M}$ be a weight vector. There is a constant $C>0$ (depending on $\Omega$ and a) such that

$$
|a(u, v)| \leqslant C|u|_{H_{\alpha}^{1,1}(\Omega)}|v|_{H_{-\boldsymbol{\alpha}}^{1,1}(\Omega)}
$$

for all $u \in \stackrel{\circ}{H}_{\boldsymbol{\alpha}}^{1,1}(\Omega), v \in \stackrel{\circ}{H}_{-\boldsymbol{\alpha}}^{1,1}(\Omega)$. Furthermore, for $f \in L^{2}(\Omega)$ and $g \in H_{\boldsymbol{\alpha}}^{1 / 2,1 / 2}(\Gamma)$, we have

$$
|\ell(v)| \leqslant C\left(\|f\|_{0, \Omega}+\|g\|_{H_{\alpha}^{1 / 2,1 / 2}(\Gamma)}\right)|v|_{H_{-\alpha}^{1,1}(\Omega)}
$$

for any $v \in \stackrel{\circ}{H}_{-\alpha}^{1,1}(\Omega)$.

Proof. There holds

$$
\begin{aligned}
|a(u, v)| & \leqslant\left\|\Phi_{\boldsymbol{\alpha}} \nabla u\right\|_{0, \Omega}\left\|\Phi_{-\boldsymbol{\alpha}} \nabla v\right\|_{0, \Omega}+\|c\|_{L^{\infty}(\Omega)}\|u\|_{0, \Omega}\|v\|_{0, \Omega} \\
& \leqslant C\left(|u|_{H_{\boldsymbol{\alpha}}^{1,1}(\Omega)}|v|_{H_{-\boldsymbol{\alpha}}^{1,1}(\Omega)}+\|u\|_{0, \Omega}\|v\|_{0, \Omega}\right) .
\end{aligned}
$$

Furthermore, using the Poincaré-Friedrichs inequality and Lemma 2.5, we get

$$
\|u\|_{0, \Omega}\|v\|_{0, \Omega} \leqslant C|u|_{H_{\boldsymbol{a}}^{1,1}(\Omega)}|v|_{1, \Omega} \leqslant C|u|_{H_{\boldsymbol{a}}^{1,1}(\Omega)}|v|_{H_{-\boldsymbol{\alpha}}^{1,1}(\Omega)}
$$

Hence,

$$
|a(u, v)| \leqslant C|u|_{H_{\alpha}^{1,1}(\Omega)}|v|_{H_{-\alpha}^{1,1}(\Omega)}
$$

Moreover, employing the previous estimate and proceeding as before to estimate the $L^{2}$-norm, we obtain

$$
|\ell(v)| \leqslant\|f\|_{0, \Omega}\|v\|_{0, \Omega}+|a(G, v)| \leqslant\|f\|_{0, \Omega}|v|_{H_{-\alpha}^{1,1}(\Omega)}+C|G|_{H_{a}^{1,1}(\Omega)}|v|_{H_{-\alpha}^{1,1}(\Omega)} .
$$

Then applying (2.11) yields the stability bound for $\ell$.

Furthermore, the following inf-sup stability holds. 
Proposition 2.9 Let $\boldsymbol{\alpha} \in[0,1)^{M}$ be a weight vector. Suppose that the weights $\alpha_{i}, i=1,2, \ldots M$, are sufficiently small so that

$$
\mu:=\frac{1}{\pi} \max _{1 \leqslant i \leqslant M} \alpha_{i} \omega_{i}<\frac{1}{2}
$$

Then there holds

$$
\inf _{0 \neq u \in \dot{H}_{\alpha}^{1,1}(\Omega)} \sup _{0 \neq v \in \dot{H}_{-\alpha}^{1,1}(\Omega)} \frac{a(u, v)}{|u|_{H_{\alpha}^{1,1}(\Omega)}|v|_{H_{-\alpha}^{1,1}(\Omega)}} \geqslant \delta,
$$

where

$$
\delta=\frac{1-2 \mu}{\sqrt{2\left(4 \mu^{2}+1\right)}} .
$$

Furthermore, we have that

$$
\sup _{u \in \stackrel{\circ}{H}_{\alpha}^{1,1}(\Omega)} a(u, v)>0 \quad \forall v \in \stackrel{\circ}{H}_{-\alpha}^{1,1}(\Omega), \quad v \not \equiv 0 .
$$

Proof. For $u \in \stackrel{\circ}{H}_{\boldsymbol{\alpha}}^{1,1}(\Omega)$ we define $\widetilde{v}=\Phi_{\boldsymbol{\alpha}}^{2} u$. Then there holds

$$
\begin{aligned}
|\widetilde{v}|_{H_{-\alpha}^{1,1}(\Omega)}^{2} & =\int_{\Omega} \Phi_{-\boldsymbol{\alpha}}^{2}|\nabla \widetilde{v}|^{2} \mathrm{~d} \boldsymbol{x} \leqslant 2 \int_{\Omega} \Phi_{\boldsymbol{\alpha}}^{-2}\left(\left|\nabla\left(\Phi_{\boldsymbol{\alpha}}^{2}\right)\right|^{2} u^{2}+\Phi_{\alpha}^{4}|\nabla u|^{2}\right) \mathrm{d} \boldsymbol{x} \\
& \leqslant 2\left(4 \int_{\Omega}\left|\nabla \Phi_{\boldsymbol{\alpha}}\right|^{2} u^{2} \mathrm{~d} \boldsymbol{x}+|u|_{H_{\alpha}^{1,1}(\Omega)}^{2}\right) .
\end{aligned}
$$

Hence, applying Lemma 2.4, results in

$$
|\widetilde{v}|_{H_{-\boldsymbol{a}}^{1,1}(\Omega)}^{2} \leqslant 2\left(4 \mu^{2}+1\right)|u|_{H_{\alpha}^{1,1}(\Omega)}^{2} .
$$

In particular, it follows that $\widetilde{v} \in H_{-\alpha}^{1,1}(\Omega)$.

Moreover, we observe that

$$
a(u, \widetilde{v})=\int_{\Omega} \nabla u \cdot \nabla \widetilde{v} \mathrm{~d} \boldsymbol{x}+\int_{\Omega} c u \widetilde{v} \mathrm{~d} \boldsymbol{x}=\int_{\Omega} \nabla u \cdot \nabla\left(\Phi_{\boldsymbol{\alpha}}^{2} u\right) \mathrm{d} \boldsymbol{x}+\int_{\Omega} c \Phi_{\boldsymbol{\alpha}}^{2} u^{2} \mathrm{~d} \boldsymbol{x} .
$$

Thus, since $c \geqslant 0$, we get

$$
\begin{aligned}
a(u, \widetilde{v}) & \geqslant \int_{\Omega}\left(\nabla u \cdot \nabla\left(\Phi_{\boldsymbol{\alpha}}^{2}\right) u+\Phi_{\boldsymbol{\alpha}}^{2}|\nabla u|^{2}\right) \mathrm{d} \boldsymbol{x} \\
& =2 \int_{\Omega} \Phi_{\boldsymbol{\alpha}} \nabla u \cdot \nabla\left(\Phi_{\boldsymbol{\alpha}}\right) u \mathrm{~d} \boldsymbol{x}+\int_{\Omega} \Phi_{\boldsymbol{\alpha}}^{2}|\nabla u|^{2} \mathrm{~d} \boldsymbol{x} \\
& \geqslant-\frac{1}{\mu} \int_{\Omega}\left|\nabla\left(\Phi_{\boldsymbol{\alpha}}\right)\right|^{2} u^{2} \mathrm{~d} \boldsymbol{x}+(1-\mu) \int_{\Omega} \Phi_{\boldsymbol{\alpha}}^{2}|\nabla u|^{2} \mathrm{~d} \boldsymbol{x}
\end{aligned}
$$


Recalling Lemma 2.4 leads to

$$
a(u, \widetilde{v}) \geqslant-\mu|u|_{H_{a}^{1,1}(\Omega)}^{2}+(1-\mu)|u|_{H_{\alpha}^{1,1}(\Omega)}^{2} \geqslant(1-2 \mu)|u|_{H_{a}^{1,1}(\Omega)}^{2} .
$$

Now, combining (2.14) and (2.15), it follows that

$$
\sup _{v \in \dot{H}_{-\boldsymbol{\alpha}}^{1,1}(\Omega)} \frac{a(u, v)}{|u|_{H_{\alpha}^{1,1}(\Omega)}|v|_{H_{-\boldsymbol{\alpha}}^{1,1}(\Omega)}} \geqslant \frac{|u|_{H_{\boldsymbol{\alpha}}^{1,1}(\Omega)}}{|\widetilde{v}|_{H_{-\boldsymbol{\alpha}}^{1,1}(\Omega)}} \frac{a(u, \widetilde{v})}{|u|_{H_{\boldsymbol{\alpha}}^{1,1}(\Omega)}^{2}} \geqslant \delta
$$

for any $u \in \stackrel{\circ}{H}_{\alpha}^{1,1}(\Omega), u \not \equiv 0$. Taking the infimum over all $u \in \stackrel{\circ}{H}_{\boldsymbol{\alpha}}^{1,1}(\Omega)$ results in (2.12).

In addition, let $v \in \stackrel{\circ}{H}_{-\alpha}^{1,1}(\Omega), v \not \equiv 0$. Then

$$
\sup _{u \in \dot{H}_{\alpha}^{1,1}(\Omega)} a(u, v) \geqslant a(v, v) \geqslant \int_{\Omega}|\nabla v|^{2} \mathrm{~d} \boldsymbol{x} .
$$

Due to $\left.v\right|_{\Gamma}=0$ and $v \not \equiv 0$, there holds $\|\nabla v\|_{0, \Omega}>0$, and hence (2.13) holds.

The above results, Propositions 2.8 and 2.9, imply the well-posedness of the variational formulation (2.10); cf., e.g., Schwab (1998, Theorem 1.15).

THEOREM 2.10 Let $\boldsymbol{\alpha} \in[0,1)^{M}$ be a weight vector, with $\alpha_{i}, i=1,2, \ldots, M$, sufficiently small such that

$$
\max _{1 \leqslant i \leqslant M} \alpha_{i} \omega_{i}<\frac{\pi}{2}
$$

is satisfied. Furthermore, suppose that $g \in H_{\boldsymbol{\alpha}}^{1 / 2,1 / 2}(\Gamma)$ and $f \in L^{2}(\Omega)$ in (1.1)-(1.2). Then there exists exactly one solution of the weak formulation (2.10) in $H_{\alpha}^{1,1}(\Omega)$.

\section{Numerical approximation}

We shall now discuss the numerical approximation of the problem (1.1)-(1.2). To this end, we will consider $h p$-version interior penalty DG finite element methods. Particularly, we will derive an (enhanced) $L^{2}$-norm a posteriori error estimate that can be applied for adaptive purposes.

\subsection{Meshes, spaces and element edge operators}

We consider shape-regular meshes $\mathscr{T}_{h}$ that partition $\Omega \subset \mathbb{R}^{2}$ into open disjoint triangles and/or parallelograms $\{K\}_{K \in \mathscr{T}_{h}}$, i.e., $\bar{\Omega}=\bigcup_{K \in \mathscr{T}_{h}} \bar{K}$. Each element $K \in \mathscr{T}_{h}$ can then be affinely mapped onto the reference triangle $\widehat{T}=\{(\widehat{x}, \widehat{y}):-1<\widehat{x}<1,-1<\widehat{y}<-\widehat{x}\}$ or the reference square $\widehat{S}=(-1,1)^{2}$, respectively. We allow the meshes to be 1-irregular, i.e., elements may contain hanging nodes. By $h_{K}$, we denote the diameter of an element $K \in \mathscr{T}_{h}$. We assume that these quantities are of bounded variation, i.e., there is a constant $\rho_{1} \geqslant 1$ such that

$$
\rho_{1}^{-1} \leqslant h_{K_{\sharp}} / h_{K_{b}} \leqslant \rho_{1},
$$

whenever $K_{\sharp}$ and $K_{b}$ share a common edge. We store the elemental diameters in a vector $\boldsymbol{h}$ given by $\boldsymbol{h}=\left\{h_{K}: K \in \mathscr{T}_{h}\right\}$. Similarly, to each element $K \in \mathscr{T}_{h}$ we assign a polynomial degree $p_{K} \geqslant 1$ and 
define the degree vector $\boldsymbol{p}=\left\{p_{K}: K \in \mathscr{T}_{h}\right\}$. We suppose that $\boldsymbol{p}$ is also of bounded variation, i.e., there is a constant $\rho_{2} \geqslant 1$ such that

$$
\rho_{2}^{-1} \leqslant p_{K_{\sharp}} / p_{K_{b}} \leqslant \rho_{2},
$$

whenever $K_{\sharp}$ and $K_{b}$ share a common edge.

Moreover, we shall define some suitable element edge operators that are required for the DG method. To this end, we denote by $\mathscr{E} \mathscr{I}$ the set of all interior edges of the partition $\mathscr{T}_{h}$ of $\Omega$ and by $\mathscr{E}_{\mathscr{B}}$ the set of all boundary edges of $\mathscr{T}_{h}$. In addition, let $\mathscr{E}=\mathscr{E}_{\mathscr{I}} \cup \mathscr{E}_{\mathscr{B}}$. The boundary $\partial K$ of an element $K$ and the sets $\partial K \backslash \Gamma$ and $\partial K \cap \Gamma$ will be identified in a natural way with the corresponding subsets of $\mathscr{E}$.

Let $K_{\sharp}$ and $K_{b}$ be two adjacent elements of $\mathscr{T}_{h}, \partial K_{\sharp} \cap \partial K_{b}=e$ for some $e \in \mathscr{E}_{\mathcal{I}}$ and $\boldsymbol{x}$ an arbitrary point on $e$. Furthermore, let $v$ and $\boldsymbol{q}$ be scalar- and vector-valued functions, respectively, that are sufficiently smooth inside each element $K_{\sharp / b}$. By $\left(v_{\sharp / b}, \boldsymbol{q}_{\sharp / b}\right)$ we denote the traces of $(v, \boldsymbol{q})$ on $e$ taken from within the interior of $K_{\sharp / b}$, respectively. Then the averages of $v$ and $\boldsymbol{q}$ at $\boldsymbol{x} \in e$ are given by

$$
\langle v\rangle=\frac{1}{2}\left(v_{\sharp}+v_{b}\right), \quad\langle\boldsymbol{q}\rangle=\frac{1}{2}\left(\boldsymbol{q}_{\sharp}+\boldsymbol{q}_{b}\right),
$$

respectively. Similarly, the jumps of $v$ and $\boldsymbol{q}$ at $\boldsymbol{x} \in e$ are given by

$$
\llbracket v \rrbracket=v_{\sharp} \boldsymbol{n}_{K_{\sharp}}+v_{b} \boldsymbol{n}_{K_{b}}, \quad \llbracket \boldsymbol{q} \rrbracket=\boldsymbol{q}_{\sharp} \cdot \boldsymbol{n}_{K_{\sharp}}+\boldsymbol{q}_{b} \cdot \boldsymbol{n}_{K_{b}},
$$

respectively, where we denote by $\boldsymbol{n}_{K_{\sharp / b}}$ the unit outward normal vector on $\partial K_{\sharp / b}$, respectively. On a boundary edge $e \in \mathscr{E}_{\mathscr{B}}$, we set $\langle v\rangle=v,\langle\boldsymbol{q}\rangle=\boldsymbol{q}, \llbracket v \rrbracket=v \boldsymbol{n}$ and $\llbracket \boldsymbol{q} \rrbracket=\boldsymbol{q} \cdot \boldsymbol{n}$, with $\boldsymbol{n}$ denoting the unit outward normal vector on the boundary $\Gamma$.

Given a finite element mesh $\mathscr{T}_{h}$ and an associated polynomial degree vector $\boldsymbol{p}=\left(p_{K}\right)_{K \in \mathscr{T}_{h}}$, with $p_{K} \geqslant 1$ for all $K \in \mathscr{T}_{h}$, consider the $h p$-discretization space

$$
V_{\mathrm{DG}}\left(\mathscr{T}_{h}, \boldsymbol{p}\right)=\left\{v \in L^{2}(\Omega):\left.v\right|_{K} \in \mathbb{S}_{p_{K}}(K), K \in \mathscr{T}_{h}\right\}
$$

for the DG method. Here, for $K \in \mathscr{T}_{h}, \mathbb{S}_{p_{K}}(K)$ is either the space $\mathbb{P}_{p_{K}}(K)$ of all polynomials of total degree at most $p_{K}$ on $K$ or the space $\mathbb{Q}_{p_{K}}(K)$ of all polynomials of degree at most $p_{K}$ in each coordinate direction on $K$.

Finally, let us introduce the enhanced $L^{2}$-norm

$$
\|v\|_{0, h, \Omega}^{2}:=\|v\|_{0, \Omega}^{2}+\int_{\mathscr{E}} \mathrm{hp}|\llbracket v \rrbracket|^{2} \mathrm{~d} s
$$

for any $v \in H_{\boldsymbol{\alpha}}^{1,1}(\Omega)+V_{\mathrm{DG}}\left(\mathscr{T}_{h}, \boldsymbol{p}\right), \boldsymbol{\alpha} \in[0,1)^{M}$. Here the two functions $\mathrm{h} \in L^{\infty}(\mathscr{E})$ and $\mathrm{p} \in L^{\infty}(\mathscr{E})$ are given by

$$
\begin{aligned}
& \mathrm{h}(\boldsymbol{x})= \begin{cases}\min \left(h_{K_{\sharp}}, h_{K_{b}}\right) & \text { for } \boldsymbol{x} \in \partial K_{\sharp} \cap \partial K_{b} \in \mathscr{E} \mathscr{I}, \\
h_{K} & \text { for } \boldsymbol{x} \in \partial K \cap \partial \Omega \in \mathscr{E}_{\mathscr{B}},\end{cases} \\
& \mathrm{p}(\boldsymbol{x})= \begin{cases}\max \left(p_{K_{\sharp}}, p_{K_{b}}\right) & \text { for } \boldsymbol{x} \in \partial K_{\sharp} \cap \partial K_{b} \in \mathscr{E}_{\mathscr{I}}, \\
p_{K} & \text { for } \boldsymbol{x} \in \partial K \cap \partial \Omega \in \mathscr{E}_{\mathscr{B}},\end{cases}
\end{aligned}
$$

respectively. 


\section{2 hp-DG discretization}

We will now consider the following $h p$-DG formulation for the numerical approximation of (1.1)-(1.2): find $u_{\mathrm{DG}} \in V_{\mathrm{DG}}\left(\mathscr{T}_{h}, \boldsymbol{p}\right)$ such that

$$
a_{\mathrm{DG}}\left(u_{\mathrm{DG}}, v\right)=\ell_{\mathrm{DG}}(v) \quad \forall v \in V_{\mathrm{DG}}\left(\mathscr{T}_{h}, \boldsymbol{p}\right)
$$

Here

$$
\begin{aligned}
a_{\mathrm{DG}}(w, v)= & \int_{\Omega}\left(\nabla_{h} w \cdot \nabla_{h} v+c w v\right) \mathrm{d} \boldsymbol{x}-\int_{\mathscr{E}}\left\langle\nabla_{h} w \rrbracket \cdot \llbracket v \rrbracket \mathrm{d} s\right. \\
& -\int_{\mathscr{E}} \llbracket w \rrbracket \cdot\left\langle\nabla_{h} v\right\rangle \mathrm{d} s+\gamma \int_{\mathscr{E}} \sigma \llbracket w \rrbracket \cdot \llbracket v \rrbracket \mathrm{d} s
\end{aligned}
$$

is an $h p$-version symmetric interior penalty DG form, and

$$
\ell_{\mathrm{DG}}(v)=\int_{\Omega} f v \mathrm{~d} \boldsymbol{x}-\int_{\mathscr{E}_{\mathscr{B}}}\left(\nabla_{h} v \cdot \boldsymbol{n}\right) g \mathrm{~d} s+\gamma \int_{\mathscr{E}_{\mathscr{B}}} \sigma g v \mathrm{~d} s .
$$

In these forms $\nabla_{h}$ denotes the elementwise gradient operator, $\gamma>0$ is a stability constant, and the function $\sigma$ is defined by

$$
\sigma=\frac{\mathrm{p}^{2}}{\mathrm{~h}} .
$$

REMARK 3.1 Provided that $\gamma>0$ is chosen sufficiently large (independently of the local element sizes and polynomial degrees), it is well known that the DG form $a_{\mathrm{DG}}$ is coercive. More precisely, there is a constant $C>0$ independent of $\mathscr{T}_{h}$ and $\boldsymbol{p}$ such that

$$
a_{\mathrm{DG}}(v, v) \geqslant C\left(\left\|\nabla_{h} v\right\|_{0, \Omega}^{2}+\gamma \int_{\mathscr{E}} \sigma|\llbracket v \rrbracket|^{2} \mathrm{~d} s\right)
$$

for any $v \in V_{\mathrm{DG}}\left(\mathscr{T}_{h}, \boldsymbol{p}\right)$. In particular, the DG method (3.5) admits a unique solution $u_{\mathrm{DG}} \in V_{\mathrm{DG}}\left(\mathscr{T}_{h}, \boldsymbol{p}\right)$; see, e.g., Stamm \& Wihler (2010) and the references therein.

\subsection{A posteriori error estimation in the $L^{2}$-norm}

We shall now derive upper and local lower residual-based $h p$-a posteriori error estimates in the enhanced $L^{2}$-norm from (3.4) for the DG formulation (3.5).

3.3.1 Upper bound. Let us consider the dual problem

$$
\begin{aligned}
-\Delta \phi+c \phi & =e_{\mathrm{DG}} \quad \text { in } \Omega, \\
\phi & =0 \quad \text { on } \Gamma .
\end{aligned}
$$

Here

$$
e_{\mathrm{DG}}=u-u_{\mathrm{DG}}
$$


denotes the error, where $u \in H_{\boldsymbol{\alpha}}^{1,1}(\Omega), \boldsymbol{\alpha} \in[0,1)^{M}$, is the weak solution of (1.1)-(1.2) defined in (2.10), and $u_{\mathrm{DG}} \in V_{\mathrm{DG}}\left(\mathscr{T}_{h}, \boldsymbol{p}\right)$ is the DG solution defined in (3.5). Throughout this section we suppose that this problem has a solution $\phi \in H^{2}(\Omega) \cap \stackrel{\circ}{H}^{1}(\Omega)$ with continuous dependence on the data, i.e., there exists a constant $C>0$ such that

$$
\|\phi\|_{H^{2}(\Omega)} \leqslant C\left\|e_{\mathrm{DG}}\right\|_{0, \Omega}
$$

This is the case, for example, if $\Omega$ is a convex polygon since then $\Delta: H^{2}(\Omega) \cap \stackrel{\circ}{H}^{1}(\Omega) \rightarrow L^{2}(\Omega)$ is an isomorphism; cf. Grisvard (1985, Theorem 3.2.1.2), Babuška \& Guo (1988), Dauge (1988). Furthermore, we assume that the Dirichlet boundary data satisfies

$$
g=\left.u\right|_{\Gamma} \in H_{\boldsymbol{\alpha}}^{1 / 2,1 / 2}(\Gamma)
$$

Then if $\boldsymbol{\alpha} \in[0,1 / 2]^{M}$, the embedding $H_{\boldsymbol{\alpha}}^{1 / 2,1 / 2}(\Gamma) \hookrightarrow L^{2}(\Gamma)$ is continuous (this follows from Kufner 1985, Theorem 9.15), and hence, $g \in L^{2}(\Gamma)$.

We start the development of the (enhanced) $L^{2}$-norm a posteriori error estimate by writing

$$
\left\|e_{\mathrm{DG}}\right\|_{0, \Omega}^{2}=\int_{\Omega}(-\Delta \phi+c \phi) e_{\mathrm{DG}} \mathrm{d} \boldsymbol{x}=\int_{\Omega}(-\Delta \phi+c \phi) u \mathrm{~d} \boldsymbol{x}-\int_{\Omega}(-\Delta \phi+c \phi) u_{\mathrm{DG}} \mathrm{d} \boldsymbol{x} .
$$

Applying Lemma 2.6 in the first integral and integrating by parts elementwise in the second integral, noting that $\llbracket \nabla \phi \rrbracket=0$ on $\mathscr{E} \mathscr{I}$ (since $\phi \in H^{2}(\Omega)$ ), results in

$$
\begin{aligned}
\left\|e_{\mathrm{DG}}\right\|_{0, \Omega}^{2}= & \int_{\Omega}(\nabla u \cdot \nabla \phi+c u \phi) \mathrm{d} \boldsymbol{x}-\int_{\Omega}\left(\nabla_{h} u_{\mathrm{DG}} \cdot \nabla \phi+c u_{\mathrm{DG}} \phi\right) \mathrm{d} \boldsymbol{x} \\
& +\int_{\mathscr{E} \mathscr{I}} \nabla \phi \cdot \llbracket u_{\mathrm{DG}} \rrbracket \mathrm{d} s-\int_{\mathscr{E} \mathscr{B}}(\nabla \phi \cdot \boldsymbol{n})\left(u-u_{\mathrm{DG}}\right) \mathrm{d} s \\
= & \int_{\Omega} f \phi \mathrm{d} \boldsymbol{x}-\int_{\Omega}\left(\nabla_{h} u_{\mathrm{DG}} \cdot \nabla \phi+c u_{\mathrm{DG}} \phi\right) \mathrm{d} \boldsymbol{x} \\
& +\int_{\mathscr{E}_{\mathscr{I}}}\langle\nabla \phi\rangle \cdot \llbracket u_{\mathrm{DG}} \rrbracket \mathrm{d} s-\int_{\mathscr{E}_{\mathscr{B}}}(\nabla \phi \cdot \boldsymbol{n})\left(g-u_{\mathrm{DG}}\right) \mathrm{d} s .
\end{aligned}
$$

Moreover, for an arbitrary function $\phi_{h} \in V_{\mathrm{DG}}\left(\mathscr{T}_{h}, \boldsymbol{p}\right)$, exploiting (3.5) with $v=\phi_{h}$, gives

$$
\begin{aligned}
\left\|e_{\mathrm{DG}}\right\|_{0, \Omega}^{2}= & \int_{\Omega} f\left(\phi-\phi_{h}\right) \mathrm{d} \boldsymbol{x}-\int_{\Omega}\left(\nabla_{h} u_{\mathrm{DG}} \cdot \nabla_{h}\left(\phi-\phi_{h}\right)+c u_{\mathrm{DG}}\left(\phi-\phi_{h}\right)\right) \mathrm{d} \boldsymbol{x} \\
& +\int_{\mathscr{E} \mathscr{I}}\langle\nabla \phi\rangle \cdot \llbracket u_{\mathrm{DG}} \rrbracket \mathrm{d} s-\int_{\mathscr{E} \mathscr{B}}(\nabla \phi \cdot \boldsymbol{n})\left(g-u_{\mathrm{DG}}\right) \mathrm{d} s \\
& +\int_{\mathscr{E} \mathscr{B}}\left(\nabla_{h} \phi_{h} \cdot \boldsymbol{n}\right) g \mathrm{~d} s-\gamma \int_{\mathscr{E} \mathscr{B}} \sigma g \phi_{h} \mathrm{~d} s-\int_{\mathscr{E}}\left\langle\nabla_{h} u_{\mathrm{DG}}\right\rangle \cdot \llbracket \phi_{h} \rrbracket \mathrm{d} s \\
& -\int_{\mathscr{E}}\left\langle\nabla_{h} \phi_{h}\right\rangle \cdot \llbracket u_{\mathrm{DG}} \rrbracket \mathrm{d} s+\gamma \int_{\mathscr{E}} \sigma \llbracket u_{\mathrm{DG}} \rrbracket \cdot \llbracket \phi_{h} \rrbracket \mathrm{d} s .
\end{aligned}
$$


Using Green's formula in the second integral leads to

$$
\begin{aligned}
\int_{\Omega} \nabla_{h} u_{\mathrm{DG}} \cdot \nabla_{h}\left(\phi-\phi_{h}\right) \mathrm{d} \boldsymbol{x}= & -\int_{\Omega} \Delta_{h} u_{\mathrm{DG}}\left(\phi-\phi_{h}\right) \mathrm{d} \boldsymbol{x}+\sum_{K \in \mathscr{T}_{h}} \int_{\partial K}\left(\nabla_{h} u_{\mathrm{DG}} \cdot \boldsymbol{n}_{K}\right)\left(\phi-\phi_{h}\right) \mathrm{d} s \\
= & -\int_{\Omega} \Delta_{h} u_{\mathrm{DG}}\left(\phi-\phi_{h}\right) \mathrm{d} \boldsymbol{x}+\int_{\mathscr{E}}\left\langle\nabla_{h} u_{\mathrm{DG}}\right\rangle \cdot \llbracket \phi-\phi_{h} \rrbracket \mathrm{d} s \\
& +\int_{\mathscr{E} \mathscr{I}} \llbracket \nabla_{h} u_{\mathrm{DG}} \rrbracket\left\langle\phi-\phi_{h}\right\rangle \mathrm{d} s,
\end{aligned}
$$

where $\Delta_{h}$ is the elementwise Laplace operator. Hence, using that $\llbracket \phi \rrbracket=\mathbf{0}$ on $\mathscr{E}$, yields

$$
\begin{aligned}
\left\|e_{\mathrm{DG}}\right\|_{0, \Omega}^{2}= & \int_{\Omega}\left(f+\Delta_{h} u_{\mathrm{DG}}-c u_{\mathrm{DG}}\right)\left(\phi-\phi_{h}\right) \mathrm{d} \boldsymbol{x}-\int_{\mathscr{E} \mathscr{F}} \llbracket \nabla_{h} u_{\mathrm{DG}} \rrbracket\left\langle\phi-\phi_{h}\right\rangle \mathrm{d} s \\
& +\int_{\mathscr{E} \mathscr{F}}\langle\nabla \phi\rangle \cdot \llbracket u_{\mathrm{DG}} \rrbracket \mathrm{d} s-\int_{\mathscr{E} \mathscr{B}}(\nabla \phi \cdot \boldsymbol{n})\left(g-u_{\mathrm{DG}}\right) \mathrm{d} s+\int_{\mathscr{E} \mathscr{B}}\left(\nabla_{h} \phi_{h} \cdot \boldsymbol{n}\right) g \mathrm{~d} s \\
& -\gamma \int_{\mathscr{E} \mathscr{B}} \sigma g \phi_{h} \mathrm{~d} s-\int_{\mathscr{E}}\left\langle\nabla_{h} \phi_{h}\right\rangle \cdot \llbracket u_{\mathrm{DG}} \rrbracket \mathrm{d} s+\gamma \int_{\mathscr{E}} \sigma \llbracket u_{\mathrm{DG}} \rrbracket \cdot \llbracket \phi_{h} \rrbracket \mathrm{d} s \\
= & \int_{\Omega}\left(f+\Delta_{h} u_{\mathrm{DG}}-c u_{\mathrm{DG}}\right)\left(\phi-\phi_{h}\right) \mathrm{d} \boldsymbol{x}-\int_{\mathscr{E} \mathscr{I}} \llbracket \nabla_{h} u_{\mathrm{DG}} \rrbracket\left\langle\phi-\phi_{h}\right\rangle \mathrm{d} s \\
& +\int_{\mathscr{E} \mathscr{I}}\left\langle\nabla_{h}\left(\phi-\phi_{h}\right)\right\rangle \cdot \llbracket u_{\mathrm{DG}} \rrbracket \mathrm{d} s-\int_{\mathscr{E} \mathscr{B}}\left(\nabla_{h}\left(\phi-\phi_{h}\right) \cdot \boldsymbol{n}\right)\left(g-u_{\mathrm{DG}}\right) \mathrm{d} s \\
& -\gamma \int_{\mathscr{E} \mathscr{B}} \sigma\left(g-u_{\mathrm{DG}}\right)\left(\phi_{h}-\phi\right) \mathrm{d} s+\gamma \int_{\mathscr{E} \mathscr{I}} \sigma \llbracket u_{\mathrm{DG}} \rrbracket \cdot \llbracket \phi_{h}-\phi \rrbracket \mathrm{d} s .
\end{aligned}
$$

Now, applying the Cauchy-Schwarz inequality and noting that $p_{K} \geqslant 1, K \in \mathscr{T}_{h}$, gives

$$
\begin{aligned}
\left\|e_{\mathrm{DG}}\right\|_{0, \Omega}^{2} \leqslant & \left(\sum_{K \in \mathscr{T}_{h}} h_{K}^{4} p_{K}^{-4}\left\|f+\Delta_{h} u_{\mathrm{DG}}-c u_{\mathrm{DG}}\right\|_{0, K}^{2}+\sum_{K \in \mathscr{T}_{h}} h_{K}^{3} p_{K}^{-3}\left\|\llbracket \nabla_{h} u_{\mathrm{DG}} \rrbracket\right\|_{0, \partial K \backslash \Gamma}^{2}\right. \\
& +\left(\gamma^{2}+1\right) \sum_{K \in \mathscr{T}_{h}} h_{K} p_{K}\left\|\llbracket u_{\mathrm{DG}} \rrbracket\right\|_{0, \partial K \backslash \Gamma}^{2} \\
& \left.+\left(\gamma^{2}+1\right) \sum_{K \in \mathscr{T}_{h}} h_{K} p_{K}\left\|g-u_{\mathrm{DG}}\right\|_{0, \partial K \cap \Gamma}^{2}\right)^{\frac{1}{2}} \\
& \times\left(\sum_{K \in \mathscr{T}_{h}} h_{K}^{-4} p_{K}^{4}\left\|\phi-\phi_{h}\right\|_{0, K}^{2}+\sum_{K \in \mathscr{T}_{h}} h_{K}^{-3} p_{K}^{3}\left\|\phi-\phi_{h}\right\|_{0, \partial K}^{2}\right. \\
& \left.+\sum_{K \in \mathscr{T}_{h}} h_{K}^{-1} p_{K}\left\|\nabla_{h}\left(\phi-\phi_{h}\right)\right\|_{0, \partial K}^{2}\right)^{\frac{1}{2}} .
\end{aligned}
$$


Then choosing $\phi_{h} \in V_{\mathrm{DG}}\left(\mathscr{T}_{h}, \boldsymbol{p}\right)$ to be an elementwise optimal $h p$-interpolant (see, e.g., Babuška \& Suri, 1987a,b), i.e., for any $K \in \mathscr{T}_{h}$,

$$
h_{K}^{-4} p_{K}^{4}\left\|\phi-\phi_{h}\right\|_{0, K}^{2}+h_{K}^{-3} p_{K}^{3}\left\|\phi-\phi_{h}\right\|_{0, \partial K}^{2}+h_{K}^{-1} p_{K}\left\|\nabla_{h}\left(\phi-\phi_{h}\right)\right\|_{0, \partial K}^{2} \leqslant C\|\phi\|_{H^{2}(K)}^{2},
$$

and recalling the regularity estimate (3.12) gives

$$
\left\|e_{\mathrm{DG}}\right\|_{0, \Omega}^{2} \leqslant C\left\|e_{\mathrm{DG}}\right\|_{0, \Omega}\left(\sum_{K \in \mathscr{T}_{h}} \widetilde{\eta}_{K}^{2}\right)^{\frac{1}{2}},
$$

with

$$
\begin{aligned}
\widetilde{\eta}_{K}^{2}= & h_{K}^{4} p_{K}^{-4}\left\|f+\Delta_{h} u_{\mathrm{DG}}-c u_{\mathrm{DG}}\right\|_{0, K}^{2}+h_{K}^{3} p_{K}^{-3}\left\|\llbracket \nabla_{h} u_{\mathrm{DG}} \rrbracket\right\|_{0, \partial K \backslash \Gamma}^{2} \\
& +h_{K} p_{K}\left\|\llbracket u_{\mathrm{DG}} \rrbracket\right\|_{0, \partial K \backslash \Gamma}^{2}+h_{K} p_{K}\left\|g-u_{\mathrm{DG}}\right\|_{0, \partial K \cap \Gamma}^{2} .
\end{aligned}
$$

Hence, dividing both sides of the above inequality by $\left\|e_{\mathrm{DG}}\right\|_{0, \Omega}$ leads to

$$
\left\|e_{\mathrm{DG}}\right\|_{0, \Omega}^{2} \leqslant C \sum_{K \in \mathscr{T}_{h}} \widetilde{\eta}_{K}^{2} .
$$

Furthermore, since $u \in H_{\boldsymbol{\alpha}}^{1,1}(\Omega)$ it holds that $\llbracket u \rrbracket=0$ on all interior edges of $\mathscr{T}_{h}$, see Wihler (2002, Lemma 1.3.4). Consequently,

$$
\left\|\llbracket e_{\mathrm{DG}} \rrbracket\right\|_{0, e}=\left\|\llbracket u_{\mathrm{DG}}-u \rrbracket\right\|_{0, e}=\left\|\llbracket u \rrbracket-\llbracket u_{\mathrm{DG}} \rrbracket\right\|_{0, e}=\left\|\llbracket u_{\mathrm{DG}} \rrbracket\right\|_{0, e}
$$

for any $e \in \mathscr{E} \mathscr{I}$, and

$$
\left\|\llbracket e_{\mathrm{DG}} \rrbracket\right\|_{0, e}=\left\|\llbracket u_{\mathrm{DG}}-g \rrbracket\right\|_{0, e}
$$

if $e \in \mathscr{E}_{\mathscr{B}}$.

Hence, denoting by $\Pi_{h} f$ the elementwise $L^{2}$-projection into $V_{\mathrm{DG}}\left(\mathscr{T}_{h}, \boldsymbol{p}\right)$, we obtain the following result.

THEOREM 3.2 Suppose that the dual problem (3.9)-(3.10) fulfils (3.12), and that the Dirichlet boundary data $g \in H_{\boldsymbol{\alpha}}^{1 / 2,1 / 2}(\Gamma)$, for some weight vector $\boldsymbol{\alpha} \in[0,1 / 2]^{M}$. Furthermore, let $u_{\mathrm{DG}} \in V_{\mathrm{DG}}\left(\mathscr{T}_{h}, \boldsymbol{p}\right)$ denote the $h p$-DG solution from (3.5), and $u \in H_{\boldsymbol{\alpha}}^{1,1}(\Omega)$ the analytical weak solution of (1.1)-(1.2). Then the following a posteriori error estimate holds

$$
\left\|u-u_{\mathrm{DG}}\right\|_{0, h, \Omega}^{2} \leqslant C\left(\sum_{K \in \mathscr{T}_{h}} \eta_{K}^{2}+\sum_{K \in \mathscr{T}_{h}} h_{K}^{4} p_{K}^{-4}\left\|f-\Pi_{h} f\right\|_{0, K}^{2}\right),
$$

where the local error indicators $\eta_{K}, K \in \mathscr{T}_{h}$, are defined by

$$
\begin{aligned}
\eta_{K}^{2}= & h_{K}^{4} p_{K}^{-4}\left\|\Pi_{h} f+\Delta_{h} u_{\mathrm{DG}}-c u_{\mathrm{DG}}\right\|_{0, K}^{2}+h_{K}^{3} p_{K}^{-3}\left\|\llbracket \nabla_{h} u_{\mathrm{DG}} \rrbracket\right\|_{0, \partial K \backslash \Gamma}^{2} \\
& +h_{K} p_{K}\left\|\llbracket u_{\mathrm{DG}} \rrbracket\right\|_{0, \partial K \backslash \Gamma}^{2}+h_{K} p_{K}\left\|g-u_{\mathrm{DG}}\right\|_{0, \partial K \cap \Gamma}^{2},
\end{aligned}
$$

and $C>0$ is a constant independent of the local element sizes $\boldsymbol{h}$ and polynomial degrees $\boldsymbol{p}$.

REMARK 3.3 We point out that Theorem 3.2 provides an upper bound on the error $u-u_{\text {DG }}$ measured in terms of the enhanced $L^{2}$-norm $\|\cdot\|_{0, h, \Omega}$ from (3.4). This norm has been exploited since it allows for the derivation of local lower bounds; this topic will be addressed in the Section 3.3.2. 
3.3.2 Local lower bound. In order to derive local lower bounds we make the simplifying assumptions that the mesh $\mathscr{T}_{h}$ consists of quadrilaterals only and that the coefficient $c$ from (1.1) is elementwise constant, i.e.,

$$
\left.c\right|_{K}=c_{K} \in \mathbb{Q}_{0}(K) \quad \forall K \in \mathscr{T}_{h} .
$$

THEOREM 3.4 Let $K, K^{\prime} \in \mathscr{T}_{h}$ be any two neighbouring elements, $e=\partial K \cap \partial K^{\prime} \in \mathscr{E} \mathscr{I}$, and $\omega_{e}=\left(\bar{K} \cup \overline{K^{\prime}}\right)^{\circ}$. Furthermore, $\epsilon \in(0,1 / 2]$. Then the following local $h p$-version a posteriori lower bounds on the error $e_{\mathrm{DG}}$ from (3.11) hold:

(a) $h_{K}^{2} p_{K}^{-2}\left\|\Delta u_{\mathrm{DG}}-c_{K} u_{\mathrm{DG}}+\Pi_{h} f\right\|_{0, K} \leqslant C_{\epsilon}\left(p_{K}^{7 / 2+\epsilon}\left\|e_{\mathrm{DG}}\right\|_{0, K}+h_{K}^{2} p_{K}^{1+2 \epsilon}\left\|f-\Pi_{h} f\right\|_{0, K}\right)$;

(b) $h_{K}^{3 / 2} p_{K}^{-3 / 2}\left\|\llbracket \nabla_{h} u_{\mathrm{DG}} \rrbracket\right\|_{0, e}$

$\leqslant C_{\epsilon}\left(p_{K}^{11 / 2+2 \epsilon}\left\|e_{\mathrm{DG}}\right\|_{0, \omega_{e}}+h_{K}^{1 / 2} p_{K}^{1 / 2}\left\|\llbracket e_{\mathrm{DG}} \rrbracket\right\|_{0, e}+h_{K}^{2} p_{K}^{3+3 \epsilon}\left\|f-\Pi_{h} f\right\|_{0, \omega_{e}}\right) ;$

(c) for any $e \in \mathscr{E}_{\mathscr{B}}$ we have that

$$
p_{K}^{1 / 2} h_{K}^{1 / 2}\left\|u_{\mathrm{DG}}-g\right\|_{0, e}=p_{K}^{1 / 2} h_{K}^{1 / 2}\left\|e_{\mathrm{DG}}\right\|_{0, e}
$$

and for $e \in \mathscr{E} \mathscr{I}$ that

$$
p_{K}^{1 / 2} h_{K}^{1 / 2}\left\|\llbracket u_{\mathrm{DG}} \rrbracket\right\|_{0, e}=p_{K}^{1 / 2} h_{K}^{1 / 2}\left\|\llbracket e_{\mathrm{DG}} \rrbracket\right\|_{0, e} .
$$

Here the constant $C_{\epsilon}>0$ is independent of $\boldsymbol{h}$ and $\boldsymbol{p}$.

Before proving these estimates we introduce the following auxiliary results.

LEMMA 3.5 Let $\widehat{K}=(0,1)^{2}$ be the unit square, and $\epsilon \in(0,5 / 2]$. We define the cut-off function

$$
\widehat{B}_{\widehat{K}}^{\beta}(\widehat{x}, \widehat{y})=\widehat{x}^{\beta}(1-\widehat{x})^{\beta} \widehat{y}^{\beta}(1-\widehat{y})^{\beta},
$$

where $\beta=\frac{3}{2}+\epsilon$. Then $\widehat{B}_{\widehat{K}}^{\beta}=0$ and $\nabla \widehat{B}_{\widehat{K}}^{\beta}=\mathbf{0}$ on $\partial \widehat{K}$, and $\Delta \widehat{B}_{\widehat{K}}^{\beta} \in L^{2}(\widehat{K})$. Furthermore, for any $\widehat{v} \in \mathbb{Q}_{p}(\widehat{K}), p \geqslant 1$, we have

$$
\left\|\Delta\left(\widehat{B}_{\widehat{K}}^{\beta} \widehat{v}\right)\right\|_{0, \widehat{K}} \leqslant C_{\epsilon} p^{4-\beta}\left\|\widehat{B}_{\widehat{K}}^{\beta / 2} \widehat{v}\right\|_{0, \widehat{K}},
$$

and

$$
\|\widehat{v}\|_{0, \widehat{K}} \leqslant C_{\epsilon} p^{2 \beta}\left\|\widehat{B}_{\widehat{K}}^{\beta / 2} \widehat{v}\right\|_{0, \widehat{K}},
$$

with a constant $C_{\epsilon}>0$ independent of $p$ and of $\widehat{v}$.

Proof. The vanishing boundary value properties follow immediately from the definition of $\widehat{B}_{\widehat{K}}^{\beta}$. Furthermore, the estimates result from tensorizing corresponding one-dimensional (1D) results; see, e.g., Bernardi et al. (2001, Lemmas 4 and 5).

LEMMA 3.6 We consider the unit square $\widehat{K}=(0,1)^{2}$, and $0<\widehat{a}<1$. Furthermore, let $\widehat{v} \in \mathbb{P}_{p}(0,1)$, $p \geqslant 1$, and

$$
\widehat{b}^{\beta}(\widehat{y})=\widehat{y}^{\beta}(1-\widehat{y})^{\beta}
$$


a bubble function, with $\beta=\frac{3}{2}+\epsilon, \epsilon \in(0,1 / 2]$. Then there exists a cut-off function $\widehat{\chi} \in C^{1}(\widehat{K})$ with

$$
\left.\widehat{\chi}\right|_{\partial \widehat{K}}=0,\left.\quad \nabla \widehat{\chi}\right|_{\partial \widehat{K}}=\mathbf{0}
$$

and

$$
\left.\widehat{\chi}\right|_{\{\widehat{a}\} \times(0,1)}=\left.\widehat{b}^{\beta} \widehat{v}\right|_{(0,1)} .
$$

Furthermore, there hold the estimates

$$
\|\widehat{\chi}\|_{0, \widehat{K}} \leqslant C_{\widehat{a}, \epsilon}\left\|\widehat{b}^{\beta / 2} \widehat{v}\right\|_{0,(0,1)}, \quad\|\Delta \widehat{\chi}\|_{0, \widehat{K}} \leqslant C_{\widehat{a}, \epsilon} p^{4-\beta}\left\|\widehat{b}^{\beta / 2} \widehat{v}\right\|_{0,(0,1)},
$$

and

$$
\|\widehat{v}\|_{0,(0,1)} \leqslant C_{\widehat{a}, \epsilon} p^{\beta}\left\|\widehat{b}^{\beta / 2} \widehat{v}\right\|_{0,(0,1)}, \quad\|\nabla \widehat{\chi}\|_{0,(0,1)} \leqslant C_{\widehat{a}, \epsilon} p^{2-\beta}\left\|\widehat{b}^{\beta / 2} \widehat{v}\right\|_{0,(0,1)} .
$$

Here $C_{\widehat{a}, \epsilon}>0$ is a constant independent of $\widehat{v}$ and $p$.

Proof. On $\widehat{K}$ we define

$$
\widehat{\chi}(\widehat{x}, \widehat{y})=\widehat{b}^{\beta}(\widehat{a})^{-1} \widehat{b}^{\beta}(\widehat{x}) \widehat{b}^{\beta}(\widehat{y}) \widehat{v}(\widehat{y}) .
$$

The lemma is again proved by referring to suitable 1D results from Bernardi et al. (2001, Lemmas 4 and 5).

Proof of Theorem 3.4. We show each of the bounds (a)-(c) in a separate step.

Proof of $(a)$. For $K \in \mathscr{T}_{h}$ let us define the volume residual

$$
R_{K}=\Delta u_{\mathrm{DG}}-c_{K} u_{\mathrm{DG}}+\Pi_{h} f \in \mathbb{Q}_{p_{K}}(K) .
$$

Then, for $\beta=3 / 2+\epsilon, \epsilon \in(0,5 / 2]$, using Lemma 3.5, together with a scaling argument, there exists a cut-off function $B_{K}^{\beta}$ satisfying $\left.B_{K}^{\beta}\right|_{\partial K}=0$ and $\left.\nabla B_{K}^{\beta}\right|_{\partial K}=\mathbf{0}$, and

$$
\left\|\Delta\left(B_{K}^{\beta} R_{K}\right)\right\|_{0, K} \leqslant C_{\epsilon} h_{K}^{-2} p_{K}^{4-\beta}\left\|B_{K}^{\beta / 2} R_{K}\right\|_{0, K},
$$

and

$$
\left\|R_{K}\right\|_{0, K} \leqslant C_{\epsilon} p_{K}^{2 \beta}\left\|B_{K}^{\beta / 2} R_{K}\right\|_{0, K},
$$

with a constant $C_{\epsilon}>0$ independent of $h_{K}$ and $p_{K}$. Moreover, there holds

$$
\begin{aligned}
\left\|B_{K}^{\beta / 2} R_{K}\right\|_{0, K}^{2} & =\int_{K} B_{K}^{\beta} R_{K}\left(\Delta u_{\mathrm{DG}}-c_{K} u_{\mathrm{DG}}+\Pi_{h} f\right) \mathrm{d} \boldsymbol{x} \\
& =-\int_{K} B_{K}^{\beta} R_{K}\left(\Delta e_{\mathrm{DG}}-c_{K} e_{\mathrm{DG}}+f-\Pi_{h} f\right) \mathrm{d} \boldsymbol{x} .
\end{aligned}
$$

Note that since $\Delta e_{\mathrm{DG}}=\Delta u-\Delta u_{\mathrm{DG}}=c_{K} u-f-\Delta u_{\mathrm{DG}} \in L^{2}(K)$ we may apply Lemma 2.7 to see that

$$
-\int_{K} B_{K}^{\beta} R_{K} \Delta e_{\mathrm{DG}} \mathrm{d} x=\int_{K} \nabla\left(B_{K} R_{K}\right) \cdot \nabla e_{\mathrm{DG}} \mathrm{d} \boldsymbol{x}
$$

Here we observe that

$$
\left.\nabla\left(B_{K}^{\beta} R_{K}\right)\right|_{\partial K}=\left.\left.B_{K}^{\beta}\right|_{\partial K} \nabla R_{K}\right|_{\partial K}+\left.\left.R_{K}\right|_{\partial K} \nabla B_{K}^{\beta}\right|_{\partial K}=\mathbf{0}
$$


Hence, integrating by parts once again and recalling (3.17), yields

$$
\begin{aligned}
\left|\int_{K} B_{K}^{\beta} R_{K} \Delta e_{\mathrm{DG}} \mathrm{d} \boldsymbol{x}\right| & =\left|\int_{K} e_{\mathrm{DG}} \Delta\left(B_{K}^{\beta} R_{K}\right) \mathrm{d} \boldsymbol{x}\right| \leqslant\left\|e_{\mathrm{DG}}\right\|_{0, K}\left\|\Delta\left(B_{K}^{\beta} R_{K}\right)\right\|_{0, K} \\
& \leqslant C_{\epsilon} h_{K}^{-2} p_{K}^{4-\beta}\left\|e_{\mathrm{DG}}\right\|_{0, K}\left\|B_{K}^{\beta / 2} R_{K}\right\|_{0, K} .
\end{aligned}
$$

Moreover,

$$
\left|c_{K} \int_{K} B_{K}^{\beta} R_{K} e_{\mathrm{DG}} \mathrm{d} x\right| \leqslant\left|c_{K}\right|\left\|B_{K}^{\beta} R_{K}\right\|_{0, K}\left\|e_{\mathrm{DG}}\right\|_{0, K} \leqslant C\left\|B_{K}^{\beta / 2} R_{K}\right\|_{0, K}\left\|e_{\mathrm{DG}}\right\|_{0, K} .
$$

Similarly,

$$
\left|\int_{K} B_{K}^{\beta} R_{K}\left(\Pi_{h} f-f\right) \mathrm{d} x\right| \leqslant\left\|B_{K}^{\beta} R_{K}\right\|_{0, K}\left\|\Pi_{h} f-f\right\|_{0, K} \leqslant C\left\|B_{K}^{\beta / 2} R_{K}\right\|_{0, K}\left\|\Pi_{h} f-f\right\|_{0, K} .
$$

Therefore,

$$
\left\|B_{K}^{\beta / 2} R_{K}\right\|_{0, K}^{2} \leqslant C_{\epsilon}\left\|B_{K}^{\beta / 2} R_{K}\right\|_{0, K}\left(h_{K}^{-2} p_{K}^{4-\beta}\left\|e_{\mathrm{DG}}\right\|_{0, K}+\left\|\Pi_{h} f-f\right\|_{0, K}\right) .
$$

Dividing the above inequality by $\left\|B_{K}^{\beta / 2} R_{K}\right\|_{0, K}$ and employing (3.18) we deduce the inequality in (a).

Proof of $(b)$. We define, for any $e \in \mathscr{E} \mathscr{I}$, the edge residual

$$
E_{e}=\llbracket \nabla_{h} u_{\mathrm{DG}} \rrbracket \in \mathbb{P}_{p_{e}}(e),
$$

where $p_{e}:=\max \left(p_{K}, p_{K^{\prime}}\right)$. For simplicity, we make the assumption that $\mathscr{T}_{h}$ is a regular mesh (otherwise, the mesh may be suitably regularized, see Houston et al., 2008, Remark 3.9). In this case $\omega_{e}$ can be affinely mapped to the unit square $\widehat{K}=(0,1)^{2}$. By the same mapping the intersection $e=\left(\partial K \cap \partial K^{\prime}\right)^{\circ}$ is transformed to a unit edge $\{\widehat{a}\} \times(0,1) \subset \widehat{K}$, with $0<\widehat{a}<1$, i.e., $K$ is mapped to $(0, \widehat{a}) \times(0,1)$ and $K^{\prime}$ is mapped to $(\widehat{a}, 1) \times(0,1)$. Hence, we may apply Lemma 3.6 (with $\left.\epsilon \in(0,1 / 2]\right)$ to obtain a cut-off function $\chi_{e} \in C^{1}\left(\omega_{e}\right)$ and a bubble function $b_{e}^{\beta}$ on $e$ with

$$
\left.\chi_{e}\right|_{\partial \omega_{e}}=0,\left.\quad \nabla \chi_{e}\right|_{\partial \omega_{e}}=\mathbf{0},
$$

and

$$
\left\|\chi_{e}\right\|_{0, \omega_{e}} \leqslant C h_{K}^{1 / 2}\left\|b_{e}^{\beta / 2} E_{e}\right\|_{0, e}, \quad\left\|\Delta \chi_{e}\right\|_{0, \omega_{e}} \leqslant C h_{K}^{-3 / 2} p_{K}^{4-\beta}\left\|b_{e}^{\beta / 2} E_{e}\right\|_{0, e} .
$$

Note that, due to (3.1) and (3.2), it holds that $h_{K} \sim h_{K^{\prime}}$, and $p_{e} \sim p_{K} \sim p_{K^{\prime}}$. Moreover,

$$
\left\|E_{e}\right\|_{0, e} \leqslant C p_{K}^{\beta}\left\|b_{e}^{\beta / 2} E_{e}\right\|_{0, e}, \quad\left\|\nabla \chi_{e}\right\|_{0, e} \leqslant C h_{K}^{-1} p_{K}^{2-\beta}\left\|b_{e}^{\beta / 2} E_{e}\right\|_{0, e} .
$$

Here $C>0$ is a constant independent of $E_{e}, p_{K}$ and $p_{K^{\prime}}$. Also, we have that $\widehat{a} \sim h_{K} / h_{K}+h_{K^{\prime}}$, which, with (3.1), is bounded away from 0 and 1 (independently of $\boldsymbol{h}$ ), and hence, $C$ does not depend on the element sizes either. Furthermore, we have that

$$
\left\|b_{e}^{\beta / 2} E_{e}\right\|_{0, e}^{2}=\int_{e} \chi_{e} E_{e} \mathrm{~d} s=\int_{\partial K} \chi_{e}\left(\nabla_{h} u_{\mathrm{DG}} \cdot \boldsymbol{n}_{K}\right) \mathrm{d} s+\int_{\partial K^{\prime}} \chi_{e}\left(\nabla_{h} u_{\mathrm{DG}} \cdot \boldsymbol{n}_{K^{\prime}}\right) \mathrm{d} s .
$$


Elementwise integration by parts yields

$$
\left\|b_{e}^{\beta / 2} E_{e}\right\|_{0, e}^{2}=\int_{\omega_{e}} \nabla_{h} \chi_{e} \cdot \nabla_{h} u_{\mathrm{DG}} \mathrm{d} \boldsymbol{x}+\int_{\omega_{e}} \chi_{e} \Delta_{h} u_{\mathrm{DG}} \mathrm{d} \boldsymbol{x} .
$$

Using Lemma 2.7 and integrating by parts again we obtain that

$$
\begin{aligned}
\left\|b_{e}^{\beta / 2} E_{e}\right\|_{0, e}^{2}= & -\int_{\omega_{e}} \nabla_{h} \chi_{e} \cdot \nabla_{h} e_{\mathrm{DG}} \mathrm{d} \boldsymbol{x}-\int_{\omega_{e}} \chi_{e} \Delta_{h} e_{\mathrm{DG}} \mathrm{d} \boldsymbol{x} \\
= & \int_{\omega_{e}} e_{\mathrm{DG}} \Delta_{h} \chi_{e} \mathrm{~d} \boldsymbol{x}-\int_{e} \nabla_{h} \chi_{h} \cdot \llbracket e_{\mathrm{DG}} \rrbracket \mathrm{d} s-\int_{\omega_{e}} c \chi_{e} e_{\mathrm{DG}} \mathrm{d} \boldsymbol{x} \\
& +\int_{\omega_{e}} \chi_{e}\left(\Delta_{h} u_{\mathrm{DG}}-c u_{\mathrm{DG}}+\Pi_{h} f\right) \mathrm{d} \boldsymbol{x}+\int_{\omega_{e}} \chi_{e}\left(f-\Pi_{h} f\right) \mathrm{d} \boldsymbol{x} \\
\leqslant & C\left(\left\|e_{\mathrm{DG}}\right\|_{0, \omega_{e}}\left\|\Delta_{h} \chi_{e}\right\|_{0, \omega_{e}}+\left\|\nabla_{h} \chi_{h}\right\|_{0, e}\left\|\llbracket e_{\mathrm{DG}} \rrbracket\right\|_{0, e}+\left\|e_{\mathrm{DG}}\right\|_{0, \omega_{e}}\left\|\chi_{e}\right\|_{0, \omega_{e}}\right. \\
& \left.+\left\|\Delta_{h} u_{\mathrm{DG}}-c u_{\mathrm{DG}}+\Pi_{h} f\right\|_{0, \omega_{e}}\left\|\chi_{e}\right\|_{0, \omega_{e}}+\left\|f-\Pi_{h} f\right\|_{0, \omega_{e}}\left\|\chi_{e}\right\|_{0, \omega_{e}}\right) .
\end{aligned}
$$

Applying (3.19), (3.20) and using the bound from (a) for the elementwise volume residual results in (b).

Proof of $(c)$. This follows directly from (3.13) and (3.14).

REMARK 3.7 We note that the dependence of the lower bounds in Theorem 3.4 is suboptimal with respect to the polynomial degrees. This effect has been observed earlier in the a posteriori error analysis of $h p$-methods; see, e.g., Melenk \& Wohlmuth (2001) and Houston et al. (2007, 2008). We remark, however, that the $p$-suboptimality is less pronounced in energy norm lower bounds. We also mention the alternative approach presented in Braess et al. (2009) in the context of spectral methods.

\subsection{Numerical example}

On the rectangle $\Omega=(-1,1) \times(0,1)$ we consider the PDE problem: find $u$ such that

$$
\begin{aligned}
-\Delta u=0 & \text { in } \Omega, \\
u=g & \text { on } \Gamma .
\end{aligned}
$$

We choose the Dirichlet boundary data $g$ in such a way that the analytical solution is given by

$$
u(r, \theta)=\frac{1}{\pi} \theta,
$$

where $(r, \theta)$ denote polar coordinates in $\mathbb{R}^{2}$. Note that $g$ is smooth on $\Gamma$ except at the point $(0,0)$. Indeed, in Cartesian coordinates, we have that

$$
g(x, y=0)=\left\{\begin{array}{ll}
1 & \text { for } x<0, \\
0 & \text { for } x>0,
\end{array} \quad(x, y) \in \Gamma .\right.
$$

In addition, we remark that $u \notin H^{1}(\Omega)$. However, there holds $u \in H_{\alpha}^{1,1}(\Omega)$ for any $\alpha \in(0,1)$, where the weight function for this problem is given by $\Phi_{\alpha}(\boldsymbol{x})=|\boldsymbol{x}|^{\alpha}$. Furthermore, $u$ is analytic away from $(0,0)$ and belongs to the Babuška-Guo space (see, e.g., Babuška \& Guo, 1988)

$$
B_{\alpha}^{1}(\Omega)=\left\{v \in L^{2}(\Omega):|v|_{H_{\alpha}^{k, 1}(\Omega)} \leqslant C d^{k} k ! \quad \forall k \geqslant 1 \text {, and constants } C, d>0\right\} .
$$

With this in mind, we might therefore be able to achieve exponential convergence when $h p$-refinement is employed; cf. Schötzau \& Schwab (2001). 
Firstly, however, we investigate the practical performance of the a posteriori error estimate derived in Theorem 3.2 within an automatic $h$-version adaptive refinement procedure which is based on 1 -irregular quadrilateral elements. The $h$-adaptive meshes are constructed by marking the elements for refinement/derefinement according to the size of the local error indicators $\eta_{K}$; this is done by employing the fixed fraction strategy, with refinement and derefinement fractions set to $25 \%$ and $10 \%$, respectively.

In Fig. 1(a) we show the initial mesh and computed DG solution based on employing $p=2$, i.e., biquadratic polynomials. Furthermore, the computational mesh and DG solution are depicted in Fig. 1(b and c) after 4 and 9 adaptive refinements have been undertaken, respectively. Here we observe that the
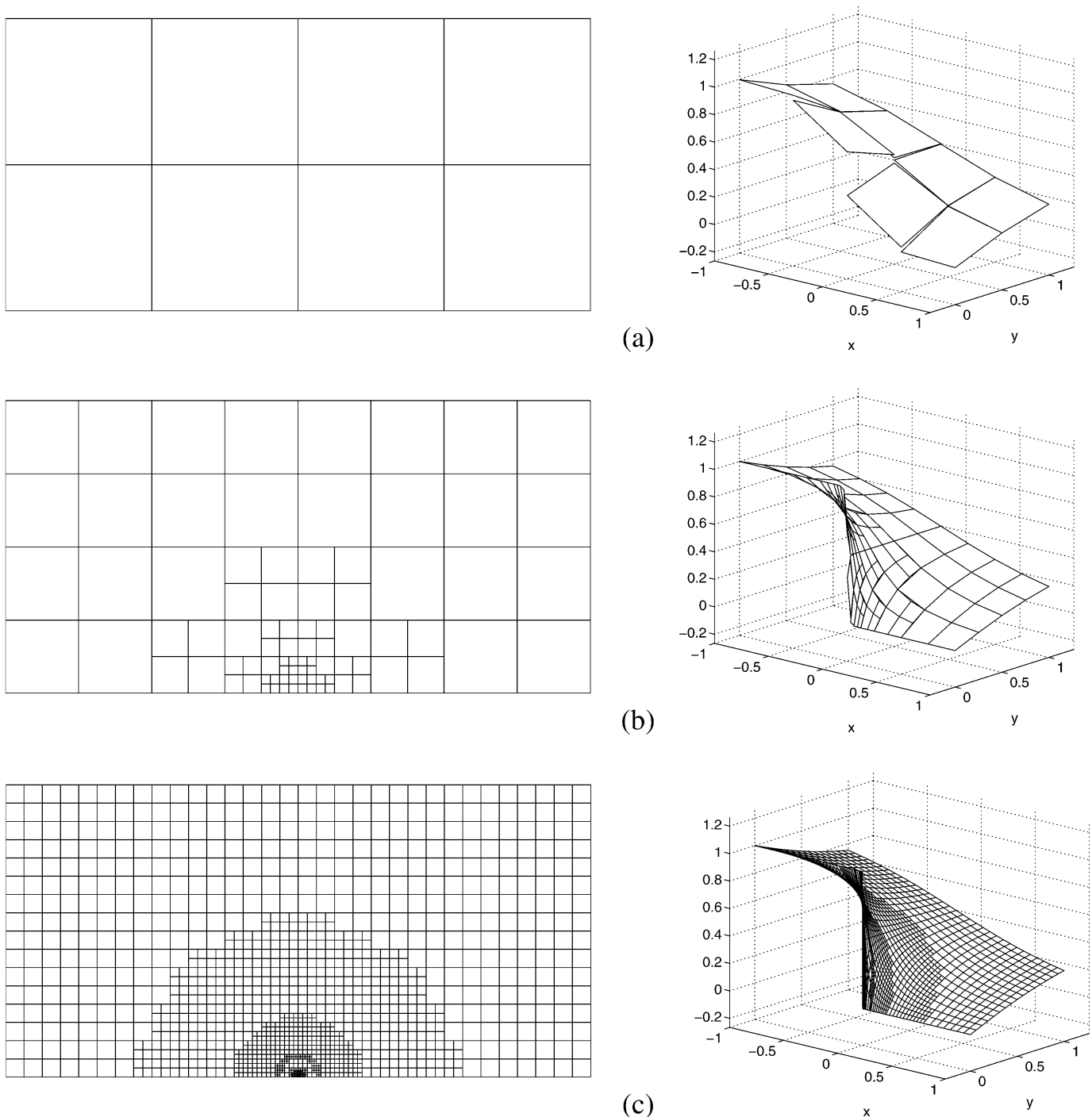

FIG. 1. $h$-Refinement. (a) Initial mesh and solution with 8 elements; mesh and solution after: (b) 4 adaptive refinements with 86 elements and (c) 9 adaptive refinements with 1286 elements. 
mesh has been significantly refined in the vicinity of the discontinuity present in $g$ as we would expect. Figure 2(a) shows the history of the actual and estimated $\|\cdot\|_{0, h, \Omega}$-norm of the error on each of the meshes generated based on employing $h$-adaptive refinement. Here we observe that the a posteriori bound over estimates the true error by a consistent factor. Indeed, the effectivity index tends to a value of around 1.5 as the mesh is adaptively refined, cf. Fig. 2(b).

We now turn our attention to $h p$-mesh adaptation. Here we again mark elements for refinement/ derefinement according to the size of the local error indicators $\eta_{K}$ based on employing the fixed fraction strategy, with refinement and derefinement fractions set to $25 \%$ and $10 \%$, respectively. Once an element $K \in \mathscr{T}_{h}$ has been flagged for refinement or derefinement, a decision must be made whether the local mesh size $h_{K}$ or the local degree $p_{K}$ of the approximating polynomial should be adjusted accordingly. The choice to perform either $h$-refinement/derefinement or $p$-refinement/derefinement is based on estimating the local smoothness of the (unknown) analytical solution. To this end, we employ the $h p$-adaptive strategy developed in Houston \& Süli (2005), where the local regularity of the analytical solution is estimated from truncated local Legendre expansions of the computed numerical solution; see, also, Houston et al. (2003).

In Fig. 3(a) we present a comparison of the actual and estimated $\|\cdot\|_{0, h, \Omega}$-norm of the error versus the third root of the number of degrees of freedom in the finite element space $V_{\mathrm{DG}}\left(\mathscr{T}_{h}, \boldsymbol{p}\right)$ on a linear$\log$ scale for the sequence of meshes generated by our $h p$-adaptive algorithm. We remark that the third root of the number of degrees of freedom is chosen on the basis of the a priori error analysis carried out in Wihler et al. (2003); cf., also, Schötzau \& Wihler (2003). Here we observe that the error bound over estimates the true error by a (reasonably) consistent factor; indeed, from Fig. 3(b), we see that the computed effectivity indices are in the range 1.5-2.5 as the mesh is refined. Moreover, from Fig. 3(a), we observe that the convergence lines using $h p$-refinement are (roughly) straight on a linear-log scale, which indicates that exponential convergence is attained for this problem.

In Fig. 4 we present a comparison between the actual error employing both $h$ - and $h p$-refinement. In particular, we compute both the enhanced $L^{2}$-norm $\left\|u-u_{\mathrm{DG}}\right\|_{0, h, \Omega}$ for which the proposed a posteriori error indicators have been derived as well as $\left\|u-u_{\mathrm{DG}}\right\|_{H_{1 / 2}^{1,1}\left(\Omega, T_{h}\right)}$. Here the norm $\|\cdot\|_{H_{1 / 2}^{1,1}\left(\Omega, T_{h}\right)}$
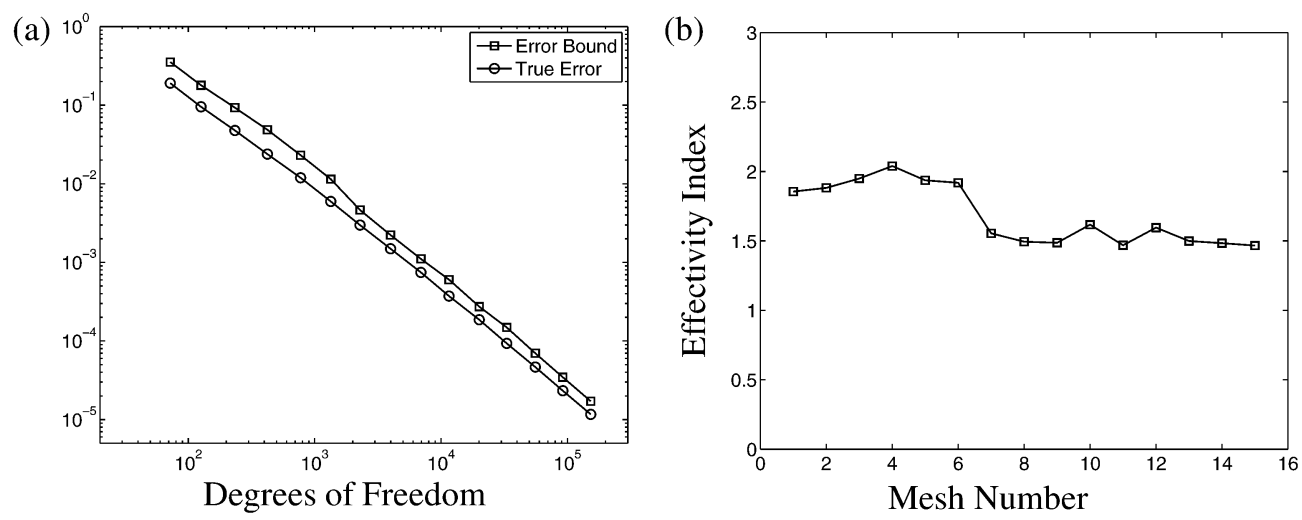

FIG. 2. $h$-Refinement. (a) Comparison of the actual and estimated $\|\cdot\|_{0, h, \Omega}$-norm of the error with respect to the number of degrees of freedom and (b) effectivity indices. 
(a)

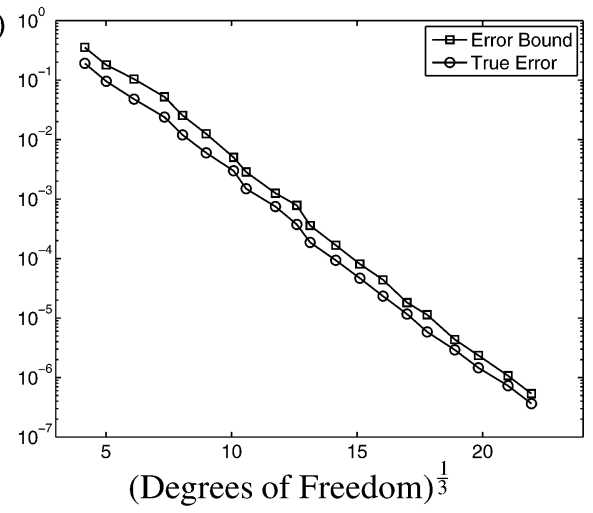

(b)

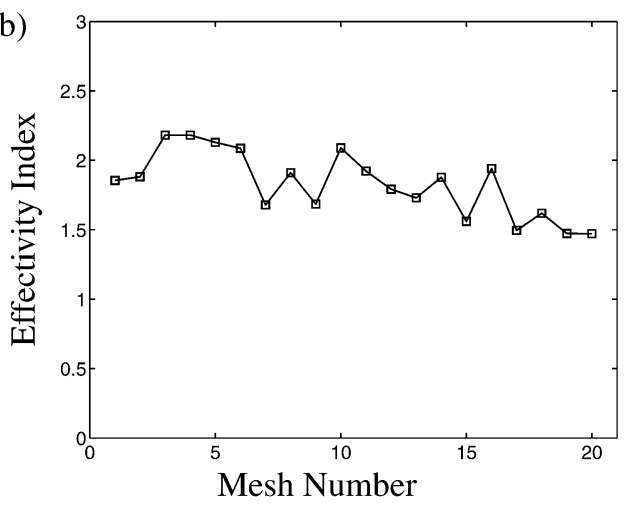

FIG. 3. $h p$-Refinement. (a) Comparison of the actual and estimated $\|\cdot\|_{0, h, \Omega}$-norm of the error with respect to the (third root of the) number of degrees of freedom and (b) effectivity indices.
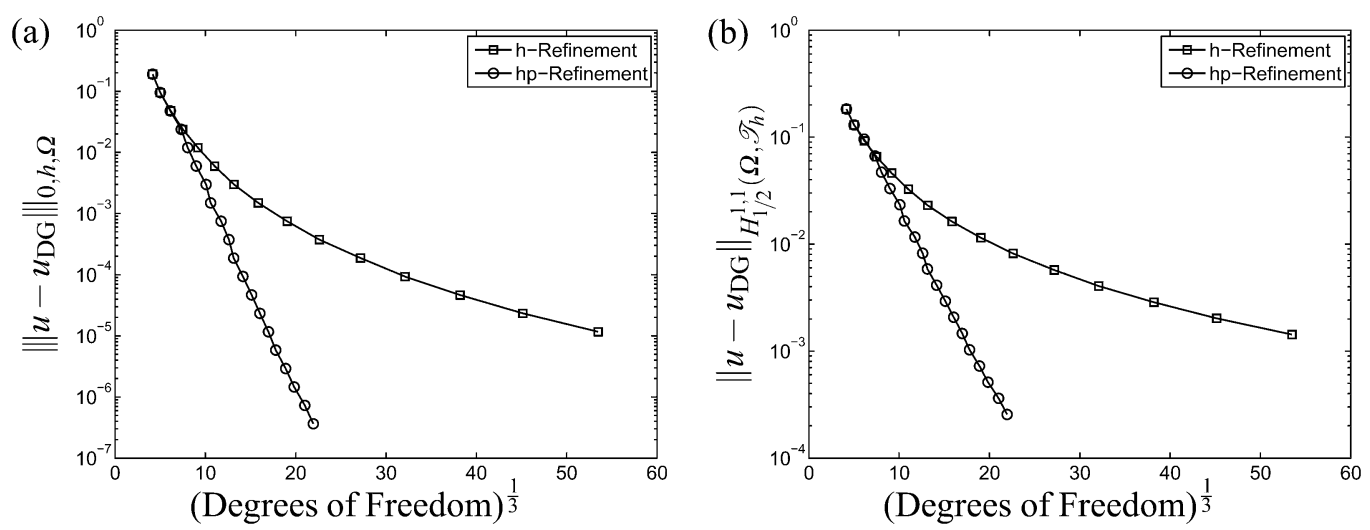

FIG. 4. Comparison between $h$ - and $h p$-refinement: (a) $\left\|u-u_{\mathrm{DG}}\right\|_{0, h, \Omega}$ and (b) $\left\|u-u_{\mathrm{DG}}\right\|_{H_{1 / 2}^{1,1}\left(\Omega, \mathscr{T}_{h}\right)}$.

represents the broken variant of the $H_{1 / 2}^{1,1}(\Omega)$-norm, i.e.,

$$
\|v\|_{H_{1 / 2}^{1,1}\left(\Omega, \mathscr{T}_{h}\right)}^{2}=\|v\|_{0, \Omega}^{2}+\left\|\Phi_{1 / 2} \nabla_{h} v\right\|_{0, \Omega}^{2}
$$

In both cases we clearly observe the superiority of employing a grid adaptation strategy based on exploiting $h p$-adaptive refinement: on the final mesh, $\left\|u-u_{\mathrm{DG}}\right\|_{0, h, \Omega}$ computed using $h p$-refinement is around three orders of magnitude smaller than the corresponding quantity computed when $h$-refinement is employed alone; an improvement of almost two orders of magnitude in $\left\|u-u_{\mathrm{DG}}\right\|_{H_{1,2}^{1,1}\left(\Omega, \mathscr{T}_{h}\right)}$ is observed when using $h p$-refinement in contrast to $h$-refinement. In addition, this demonstrates, for at least the problem at hand, that the proposed error indicator is capable of automatically guiding the adaptive process to ensure convergence of the error measured in the $H_{1 / 2}^{1,1}\left(\Omega, \mathscr{T}_{h}\right)$-norm. In general, however, a reliable upper bound on the the error computed in terms of the $H_{1 / 2}^{1,1}\left(\Omega, \mathscr{T}_{h}\right)$-norm would be necessary to ensure convergence of the adaptive algorithm, cf. below. 
Finally, in Figs. 5 and 6, we show the mesh generated using the proposed $h p$-version $a$ posteriori error indicator stated in Theorem 3.2 after 9 and $14 \mathrm{hp}$-adaptive refinement steps, respectively. For clarity, we also show the $h$-mesh alone as well as a zoom of the mesh in the vicinity of the origin. Here we observe that geometric $h$-refinement has been performed in the vicinity of the discontinuity present

(a)

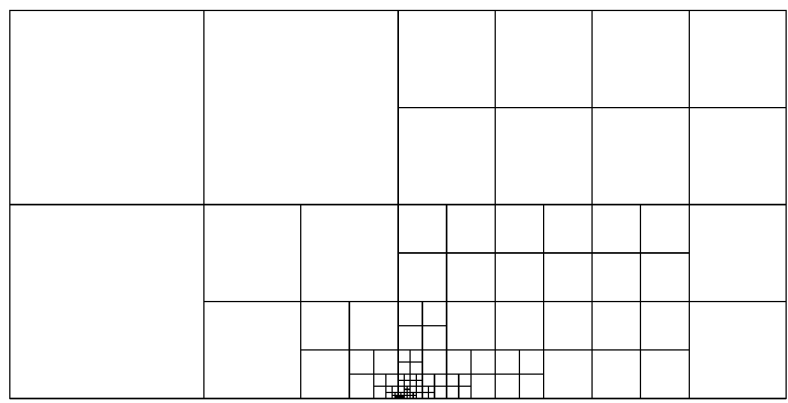

(b)

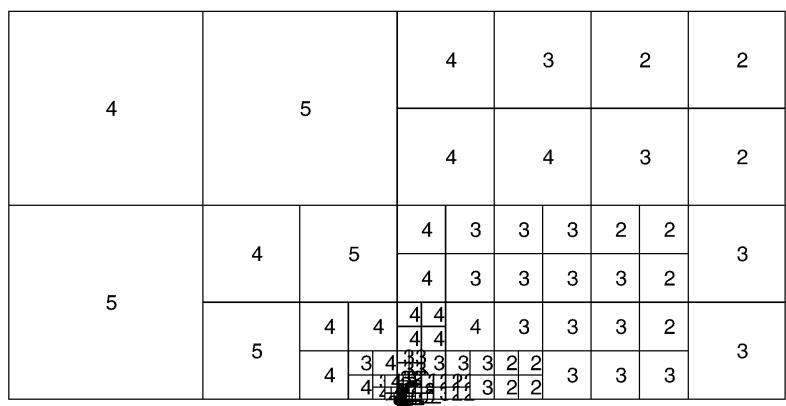

(c)

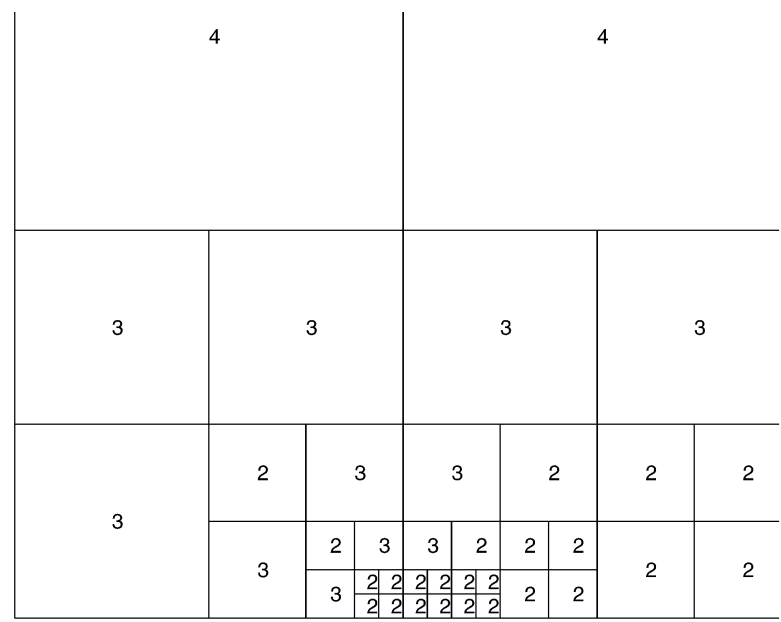

FIG. 5. $h p$-Mesh distribution after 9 adaptive refinements with 134 elements and 2002 degrees of freedom: (a) $h$-mesh alone, (b) $h p$-mesh and (c) zoom of (b). 
(a)

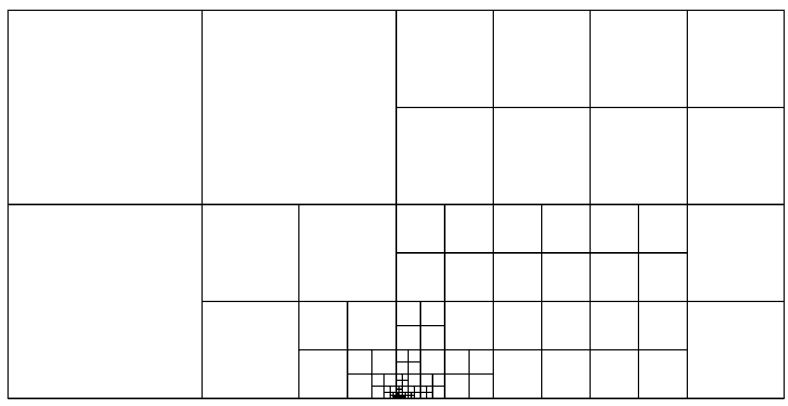

(b)

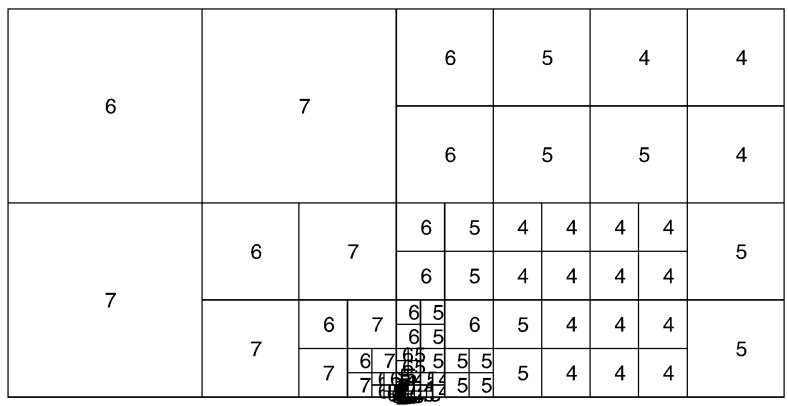

(c)

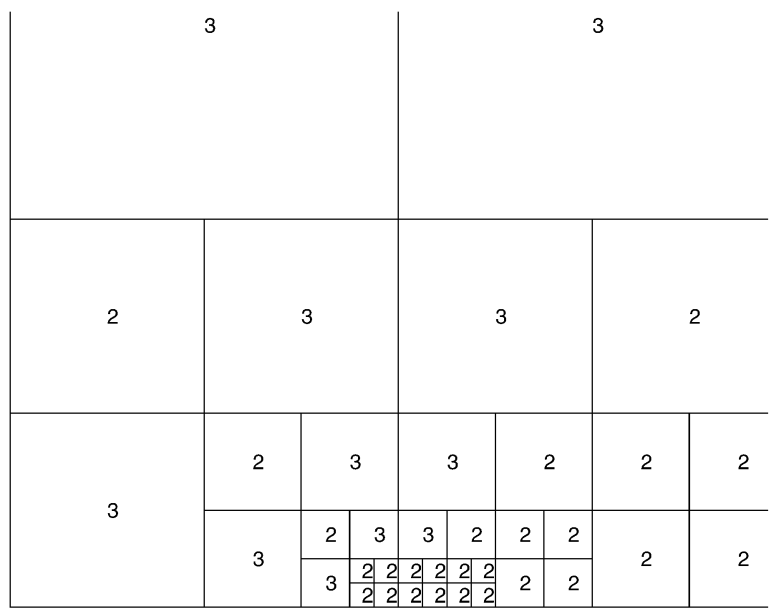

FIG. 6. $h p$-Mesh distribution after 14 adaptive refinements with 206 elements, 4904 degrees of freedom: (a) $h$-mesh alone, (b) $h p$-mesh and (c) zoom of (b).

in $g$, cf. above. Within this region, the polynomial degree has been kept at 2. Away from this region, the $h p$-adaptive algorithm increases the degree of the approximating piecewise polynomials where the analytical solution is smooth. This corresponds to the a priori $h p$-approximation strategies proposed in, e.g., Guo \& Babuška (1986a,b), Schwab (1998); see further Schwab (1998), Schötzau \& Schwab (2001), Wihler (2002), Schötzau \& Wihler (2003), Wihler et al. (2003), Houston et al. (2004). 


\section{Conclusions}

In this work we have introduced a new variational framework for linear second-order elliptic PDEs with discontinuous Dirichlet boundary conditions based on locally weighted Sobolev spaces. In particular, we have proved the well-posedness of the new setting by means of an inf-sup condition. In addition, we have proposed the use of symmetric $h p$-version interior penalty DG methods for the numerical approximation of such problems. For this discretization scheme we have derived an (enhanced) $L^{2}$-norm a posteriori error analysis featuring upper and lower estimates. The performance of the resulting error indicators within $h$ - and $h p$-adaptive refinement procedures has been displayed with a

model numerical experiment. Future work will involve an error analysis with respect to the $H_{\alpha}^{1,1}$-norm, and some extensions of the present setting to systems such as, e.g., the Stokes equations for cavity flow problems.

\section{REFERENCES}

Adams, R. A. \& Fournier, J. J. F. (2003) Sobolev Spaces, 2nd edn. Pure and Applied Mathematics Series. Amsterdam, The Netherlands: Academic Press.

ARnold, D., Brezzi, F., CockBurn, B. \& MARINI, L. (2001) Unified analysis of discontinuous Galerkin methods for elliptic problems. SIAM J. Numer. Anal., 39, 1749-1779.

BABUŠKA, I. (1971) Error bounds for finite element method. Numer. Math., 16, 322-333.

BABUŠKA, I. \& GUO, B. Q. (1988) Regularity of the solution of elliptic problems with piecewise analytic data. I. Boundary value problems for linear elliptic equation of second order. SIAM J. Math. Anal., 19, 172-203.

BABUŠKA, I. \& GUO, B. Q. (1989) Regularity of the solution of elliptic problems with piecewise analytic data. II. The trace spaces and application to the boundary value problems with nonhomogeneous boundary conditions. SIAM J. Math. Anal., 20, 763-781.

BABUŠKa, I., KellogG, R. B. \& PitKäRAnta, J. (1979) Direct and inverse error estimates for finite elements with mesh refinements. Numer. Math., 33, 447-471.

BABUŠKA, I. \& SURI, M. (1987a) The $h p$-version of the finite element method with quasiuniform meshes. RAIRO Anal. Numér., 21, 199-238.

BABUŠKA, I. \& SURI, M. (1987b) The optimal convergence rate of the $p$-version of the finite element method. SIAM J. Numer. Anal., 24, 750-776.

BAUMANN, C. \& ODEN, J. (1999) A discontinuous $h p$-finite element method for convection-diffusion problems. Comput. Methods Appl. Mech. Eng., 175, 311-341.

Bernardi, C. \& Karageorghis, A. (1999) Spectral element discretization of the circular driven cavity. I. The Laplace equation. SIAM J. Numer. Anal., 36, 1435-1465.

Bernardi, C., OWens, R. \& Valenciano, J. (2001) An error indicator for mortar element solutions to the Stokes problem. IMA J. Numer. Anal., 21, 857-886.

Braess, D., Pillwein, V. \& SchöBerl, J. (2009) Equilibrated residual error estimates are p-robust. Comput. Methods Appl. Mech. Eng., 198, 1189-1197.

Cockburn, B., Karniadakis, G. \& ShU, C.-W. (2000) The development of discontinuous Galerkin methods. Discontinuous Galerkin Methods: Theory, Computation and Applications (B. Cockburn, G. Karniadakis \& C.-W. Shu eds). Lecture Notes in Computational Science and Engineering, vol. 11. Berlin: Springer, pp. 3-50.

DAuge, M. (1988) Elliptic Boundary Value Problems on Corner Domains. Lecture Notes in Mathematics. Berlin: Springer.

Grisvard, P. (1985) Elliptic Problems in Nonsmooth Domains, Monographs and Studies in Mathematics, vol. 24. Boston, MA: Pitman (Advanced Publishing Program).

GuO, B. Q. \& BABUŠKA, I. (1986a) The $h p$-version of the finite element method. Part I: the basic approximation results. Comput. Mech., 1, 21-41. 
GuO, B. Q.\& BABUŠKA, I. (1986b) The $h p$-version of the finite element method. Part II: general results and applications. Comput. Mech., 1, 203-220.

Guo, B. \& Schwab, C. (2006) Analytic regularity of Stokes flow on polygonal domains in countably weighted Sobolev spaces. J. Comput. Appl. Math., 190, 487-519.

Hardy, G., Littlewood, J. E. \& Pólya, G. (1952) Inequalities, 2nd edn. Cambridge, UK: Cambridge University Press.

Houston, P., Schötzau, D. \& Wihler, T. P. (2004) Mixed $h p$-discontinuous Galerkin finite element methods for the Stokes problem in polygons. Numerical Mathematics and Advanced Applications (M. Feistauer, V. Dolejší, P. Knobloch, and K. Najzar eds). Berlin: Springer, pp. 493-501.

Houston, P., SchÖtZAU, D. \& WihleR, T. P. (2007) Energy norm a posteriori error estimation of $h p$-adaptive discontinuous Galerkin methods for elliptic problems. Math. Models Methods Appl. Sci., 17, 33-62.

Houston, P., Schwab, C. \& SÜLI, E. (2002) Discontinuous $h p$-finite element methods for advection-diffusionreaction problems. SIAM J. Numer. Anal., 39, 2133-2163.

Houston, P., SEnior, B. \& SÜLI, E. (2003) Sobolev regularity estimation for $h p$-adaptive finite element methods. Numerical Mathematics and Advanced Applications ENUMATH 2001 (F. Brezzi, A. Buffa, S. Corsaro \& A. Murli eds). Milano, Italia: Springer, pp. 631-656.

Houston, P. \& SÜLI, E. (2005) A note on the design of $h p$-adaptive finite element methods for elliptic partial differential equations. Comput. Methods Appl. Mech. Eng., 194, 229-243.

Houston, P., SÜLI, E. \& WiHler, T. P. (2008) A posteriori error analysis of $h p$-version discontinuous Galerkin finite-element methods for second-order quasi-linear elliptic PDEs. IMA J. Numer. Anal., 28, 245-273.

Kufner, A. (1985) Weighted Sobolev Spaces. A Wiley-Interscience Publication. New York: John Wiley \& Sons Inc., p. 116.

Melenk, J. \& Wohlmuth, B. (2001) On residual-based a posteriori error estimation in $h p$-FEM. Adv. Comput. Math., 15, 311-331.

NEČAS, J. (1962) Sur une méthode pour résoudre les équations aux dérivées partielles du type elliptique, voisine de la variationelle. Ann. Scuola Norm. Sup. Pisa, 16, 305-326.

NICAISE, S. (2000) Jacobi polynomials, weighted Sobolev spaces and approximation results of some singularities. Math. Nachr., 213, 117-140.

Perugia, I. \& SchÖtZAU, D. (2002) An $h p$-analysis of the local discontinuous Galerkin method for diffusion problems. J. Sci. Comput., 17, 561-571.

RivièRe, B., WhEELER, M. \& GiRAULT, V. (1999) Improved energy estimates for interior penalty, constrained and discontinuous Galerkin methods for elliptic problems, Part I. Comput. Geosci., 3, 337-360.

SchÖtZAU, D. \& SCHWAB, C. (2001) Exponential convergence in a Galerkin least squares $h p$-FEM for Stokes flow. IMA J. Numer. Anal., 21, 53-80.

SChÖTZAU, D. \& WiHLER, T. P. (2003) Exponential convergence of mixed $h p$-DGFEM for Stokes flow in polygons. Numer. Math., 96, 339-361.

SCHWAB, C. (1998) p- and hp-FEM-Theory and Application to Solid and Fluid Mechanics. Oxford: Oxford University Press.

StAmm, B. \& Wihler, T. P. (2010) hp-optimal discontinuous Galerkin methods for linear elliptic problems. Math. Comput., 79, 2117-2133.

Wihler, T. P. (2002) Discontinuous Galerkin FEM for elliptic problems in polygonal domains. Ph.D. thesis, Swiss Federal Institute of Technology Zurich. Diss. ETH No. 14973. Available at http://e-collection.ethbib.ethz .ch/view/eth:26201.

Wihler, T. P., Frauenfelder, P. \& Schwab, C. (2003) Exponential convergence of the $h p$-DGFEM for diffusion problems. Comput. Math. Appl., 46, 183-205. 